\title{
Zirconocene-Catalyzed Polymerization of $\alpha$-Olefins: When Intrinsic Higher Activity is Flawed by Rapid Deactivation
}

Xavier Desert, ${ }^{\mathrm{a}, \dagger}$ Fabien Proutiere, ${ }^{\mathrm{a}, \dagger}$ Alexandre Welle, ${ }^{\mathrm{b}}$ Katty Den Dauw, ${ }^{\mathrm{b}}$ Aurélien Vantomme, ${ }^{\mathrm{b}}$ Olivier Miserque, ${ }^{\mathrm{b}}$ Jean-Michel Brusson, ${ }^{\mathrm{c}}$ Jean-François Carpentier ${ }^{\mathrm{a}, \dagger^{* *}}$ and Evgueni Kirillova ${ }^{\mathrm{a},{ }^{\dagger *}}$

a Univ Rennes, CNRS, ISCR (Institut des Sciences Chimiques de Rennes), UMR 6226, F-35700 Rennes, France

b Total Research \& Technology Feluy, Zone Industrielle Feluy C, B-7181 Seneffe, Belgium

${ }^{c}$ Total S.A., Direction R\&D Groupe, Tour Michelet A, 24 Cours Michelet - La Défense 10, F-92069

Paris La Défense Cedex, France

Example of calculations of the values of ${k_{\mathrm{p}}}^{0}$ and corrected $k_{\mathrm{p}}{ }^{0}$ using the method from ref. $12 \mathrm{~d}$.

Figure S1. Polymer yield (g) as function of time (s) for the $\{\mathbf{S B I}\}-1 / \mathrm{MAO}$ system (aging time $=$ $60 \mathrm{~min} ; \mathrm{T}_{\text {aging }}=\mathrm{T}_{\text {polym }}=30^{\circ} \mathrm{C}$ ).

Figure S2. Reprocessed kinetic plots for the $\{$ SBI $\}-1 /$ MAO system from ref. 12d: polymer yield (g) as function of time (s).

Figure S3. Polymer yield (g) as function of time (s) for the $\{\mathbf{S B I}\}-2 / \mathrm{MAO}$ system (aging time $=$ $\left.60 \mathrm{~min} ; \mathrm{T}_{\text {aging }}=\mathrm{T}_{\text {polym }}=30^{\circ} \mathrm{C}\right)$.

Figure S4. Polymer yield (g) as function of time (s) for the $\{\mathbf{C p} / \mathbf{F l u}\}-\mathbf{1} / \mathrm{MAO}$ system (aging time $\left.=60 \mathrm{~min} ; \mathrm{T}_{\text {aging }}=\mathrm{T}_{\text {polym }}=30^{\circ} \mathrm{C}\right)$.

Figure S5. Polymer yield (g) as function of time (s) for the $\{\mathbf{C p} / \mathbf{F l u}\}-2 / \mathrm{MAO}$ system (aging time $\left.=60 \mathrm{~min} ; \mathrm{T}_{\text {aging }}=\mathrm{T}_{\text {polym }}=30^{\circ} \mathrm{C}\right)$.

Figure S6. Polymer yield (g) as function of time (s) for the $\{\mathbf{C p} / \mathbf{F l u}\}-3 / \mathrm{MAO}$ system (aging, 60 min; $\mathrm{T}_{\text {aging }}=\mathrm{T}_{\text {polym }}=30^{\circ} \mathrm{C}$ ).

Figure S7. Kinetic plots obtained with the $\{\mathbf{C p} / \mathbf{F l u}\}-4 / \mathrm{MAO}$ system at $30{ }^{\circ} \mathrm{C}$ and $60{ }^{\circ} \mathrm{C}$.

Figure S8. Kinetic plots obtained with the $\{\mathbf{S B I}\}-2 / \mathrm{MAO}$ system at different aging times $(60,2$ and $10 \mathrm{~min}$ ).

Figure S9. Kinetic plots obtained with the $\{\mathbf{C p} / \mathbf{F l u}\}-1 / \mathrm{MAO}$ system with variation of the aging time.

Figure S10. Kinetic plots obtained with the $\{\mathbf{C p} / \mathbf{F l u}\}-2 / \mathrm{MAO}$ system with variation of the aging time.

Figure S11. Kinetic plots obtained with the $\{\mathbf{C p} / \mathbf{F l u}\}-3 / \mathrm{MAO}$ system with variation of the aging time.

Figure S12. Kinetic plots obtained with $\{\mathbf{S B I}\}-2$ and $\{\mathbf{S B I}\}-2 \mathrm{a}$ using different coactivators.

\footnotetext{
$\uparrow$ Those two authors equally contributed to this work.

* Correspondence to Jean-François Carpentier (jean-francois.carpentier@ univ-rennes1.fr) and Evgueni Kirillov (evgueni.kirillov@univ-rennes1.fr); Fax: +33 (0)223236938.
} 
Figure S13. Kinetic plots obtained with $\{\mathbf{C p} / \mathbf{F l u}\}-\mathbf{1}$ and $\{\mathbf{C p} / \mathbf{F l u}\}-1 \mathbf{a}$ using different coactivators.

Figure S14. Kinetic plots obtained with $\{\mathbf{C p} / \mathbf{F l u}\}-3$ and $\{\mathbf{C p} / \mathbf{F l u}\}-3 a$ using different coactivators.

Figure S15. Kinetic plots obtained for $\{\mathbf{C p} / \mathbf{F l u}\}-1$ activated with BHT-modified MAO (MAO stirred for 30 min with BHT).

Figure S16. Kinetic plots obtained with the $\{\mathbf{C p} / \mathbf{F l u}\}-1 / \mathrm{MAO}$ system in the presence of 2hexene and 3-hexene.

Figure S17. Kinetic plots obtained with the $\{\mathbf{C p} / \mathbf{F l u}\}-1 / \mathrm{MAO}$ system in the presence of $\mathrm{Ph}_{2} \mathrm{SiH}_{2}$ or $\mathrm{PhSiH}_{3}$.

Figure S18. ${ }^{1} \mathrm{H}$ NMR spectrum $\left(\mathrm{C}_{6} \mathrm{D}_{6}, 400 \mathrm{MHz}, 25^{\circ} \mathrm{C}\right)$ of $\{\mathbf{C p} / \mathbf{F l u}\}-3 \mathbf{a}$.

Figure S19. ${ }^{13} \mathrm{C}\left\{{ }^{1} \mathrm{H}\right\}$ NMR spectrum $\left(\mathrm{C}_{6} \mathrm{D}_{6}, 100 \mathrm{MHz}, 25{ }^{\circ} \mathrm{C}\right)$ of $\{\mathbf{C p} / \mathbf{F l u}\}-3 a$.

Figure S20. ${ }^{1} \mathrm{H}_{-}{ }^{13} \mathrm{C}$ HSQC NMR spectrum $\left(\mathrm{C}_{6} \mathrm{D}_{6}, 400 \mathrm{MHz}, 25^{\circ} \mathrm{C}\right)$ of $\{\mathbf{C p} / \mathbf{F l u}\}-3 \mathbf{a}$.

Figure S21. ${ }^{1} \mathrm{H}_{-}{ }^{13} \mathrm{C}$ HMBC NMR spectrum $\left(\mathrm{C}_{6} \mathrm{D}_{6}, 400 \mathrm{MHz}, 25{ }^{\circ} \mathrm{C}\right)$ of $\{\mathbf{C p} / \mathbf{F l u}\}-3 \mathbf{a}$.

Figure S22. ${ }^{13} \mathrm{C}$ NMR spectrum $\left(125 \mathrm{MHz}, 135{ }^{\circ} \mathrm{C}, 1,2,4\right.$-trichlorobenzene/ $\left.\mathrm{C}_{6} \mathrm{D}_{6}\right)$ of copolymer obtained from propylene and ethylene with the $\{\mathbf{S B I}\}-2 / \mathrm{MAO}$ system.

Figure S23. The end-groups region of ${ }^{1} \mathrm{H}$ NMR spectra $\left(400 \mathrm{MHz}, 25{ }^{\circ} \mathrm{C}, \mathrm{CDCl}_{3}\right)$ of poly $(1-$ hexene)s obtained with metallocene/MAO systems in toluene (Table 2, entries 1, 3, 6 and 9, respectively). 

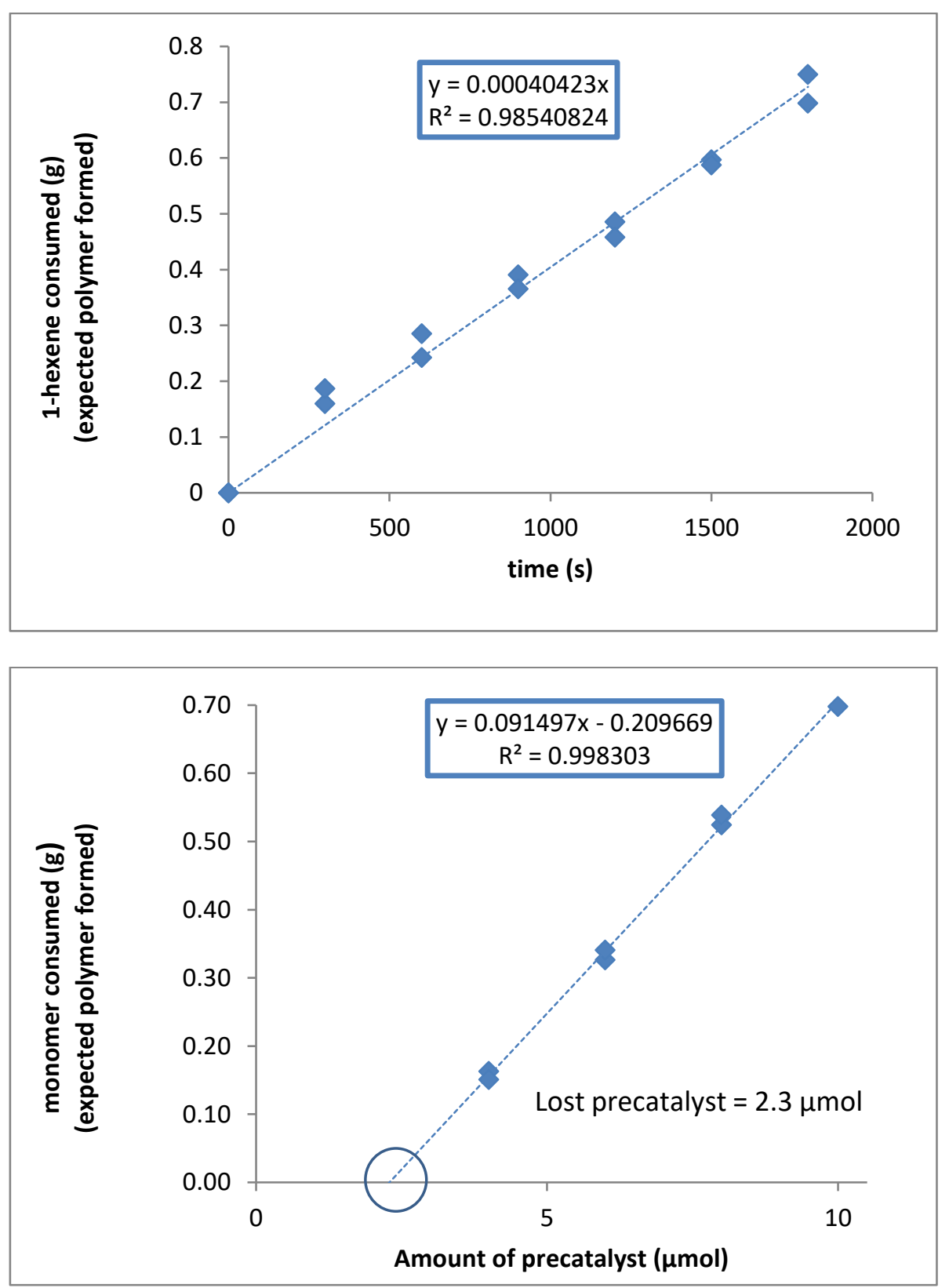

Example of calculations of the values of ${k_{\mathrm{p}}}^{0}$ and corrected $\boldsymbol{k}_{\mathrm{p}}{ }^{0}$ using the method from ref.

12d (Conditions: batch reaction, toluene, $\mathrm{T}=30^{\circ} \mathrm{C}, \mathrm{V}=50 \mathrm{~mL},[\mathrm{M}]_{0}=1.6 \mathrm{~mol} \cdot \mathrm{L}^{-1}, \mathrm{C}_{\mathrm{m}}{ }^{0}=10$ $\mu \mathrm{mol})$.

With $[\mathrm{M}]_{0}=$ initial monomer concentration, and $\mathrm{C}_{\mathrm{m}}{ }^{0}=$ amount of initially added catalyst:

Slope in Fig S1 (top) $=4.04 \times 10^{-4}$.

From equation (1) : $k_{\mathrm{p}}{ }^{0}=$ Slope $/\left([\mathrm{M}]_{0} \times \mathrm{M}_{\mathrm{M}} \times \mathrm{C}_{\mathrm{m}}{ }^{0}\right)=0.30 \mathrm{~L} \cdot \mathrm{mol} \cdot \mathrm{s}^{-1}$.

Taking into account catalyst deactivation from Fig. S1 (middle), $\mathrm{C}_{\mathrm{m}}=10-2.7=7.3 \mu \mathrm{mol}$, hence $k_{\mathrm{p}}{ }^{0}$ corr $=$ Slope $/\left([\mathrm{M}]_{0} \times \mathrm{M}_{\mathrm{M}} \times \mathrm{C}_{\mathrm{m}}\right)=(0.39 \pm 0.4) \mathrm{L} \cdot \mathrm{mol} \cdot \mathrm{s}^{-1}$. 


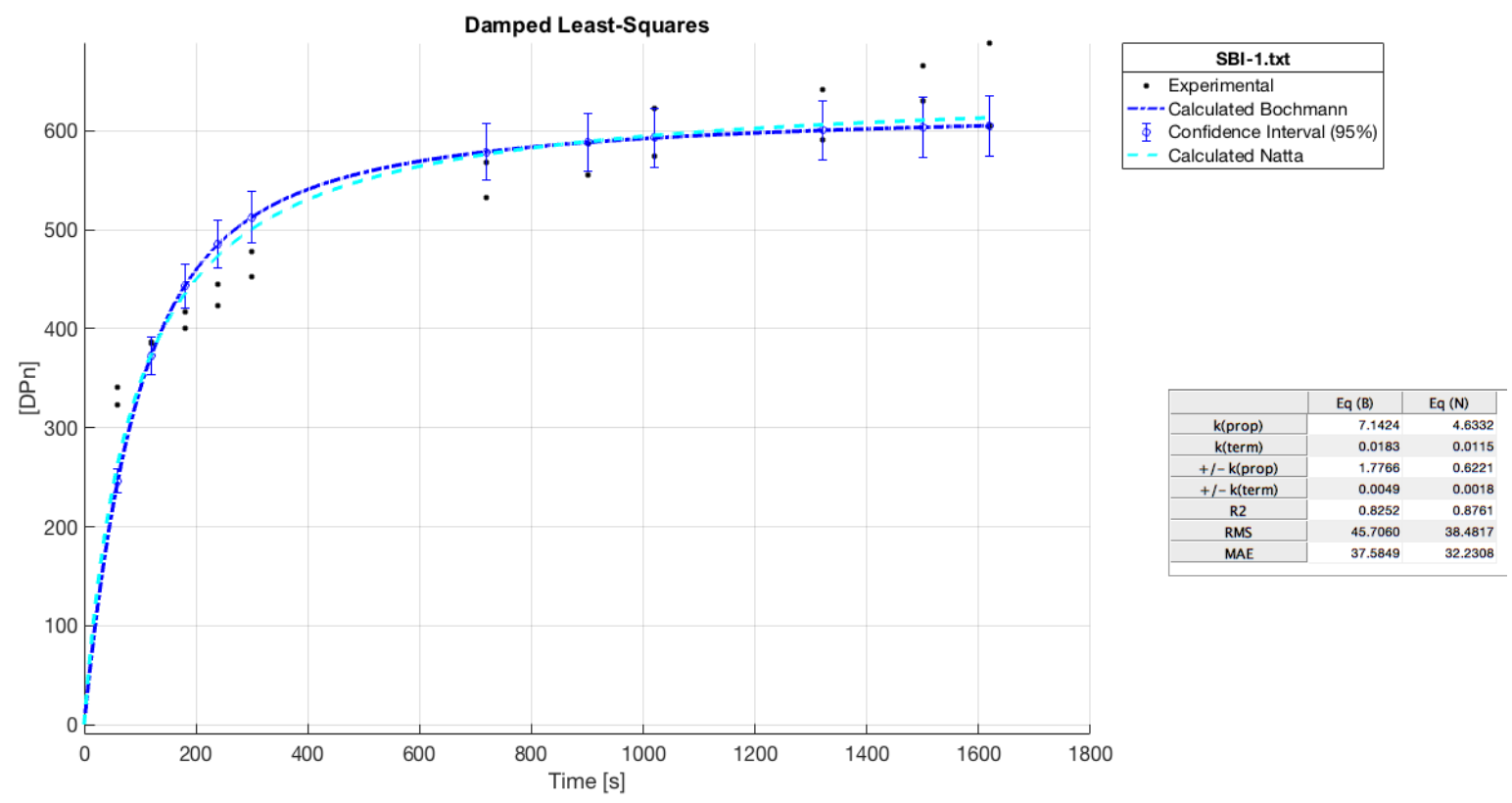

Figure S1. Kinetic plots for the $\{$ SBI $\}-1 / M A O$ system (aging time $=60 \mathrm{~min} ; \mathrm{T}_{\text {aging }}=\mathrm{T}_{\mathrm{polym}}=30$ ${ }^{\circ} \mathrm{C}$ ). Polymer yield (g) as function of time (s) (top); polymer yield (g) as function of the amount of precatalyst $(\mu \mathrm{mol}), \mathrm{t}=5 \mathrm{~min}$ (middle); evolution of polymerization degree as function of time (t) (bottom), $\mathrm{R}_{(\text {Bochmann })}=0.825, \mathrm{R}^{2}$ (Natta) $=0.876 ; \mathrm{RMS}_{(\mathrm{B})}=4.571 \cdot 10^{1}, \mathrm{RMS}_{(\mathrm{N})}=3.848 \cdot 10^{1}$. The two values of rate constants $k_{\mathrm{p}}{ }^{\mathrm{B}}$ calculated for the two independent experiments were of 0.2976 and $0.3028 \mathrm{~L} \cdot \mathrm{mol}^{-1} \cdot \mathrm{s}^{-1}(1.7 \%$ difference $)$. 

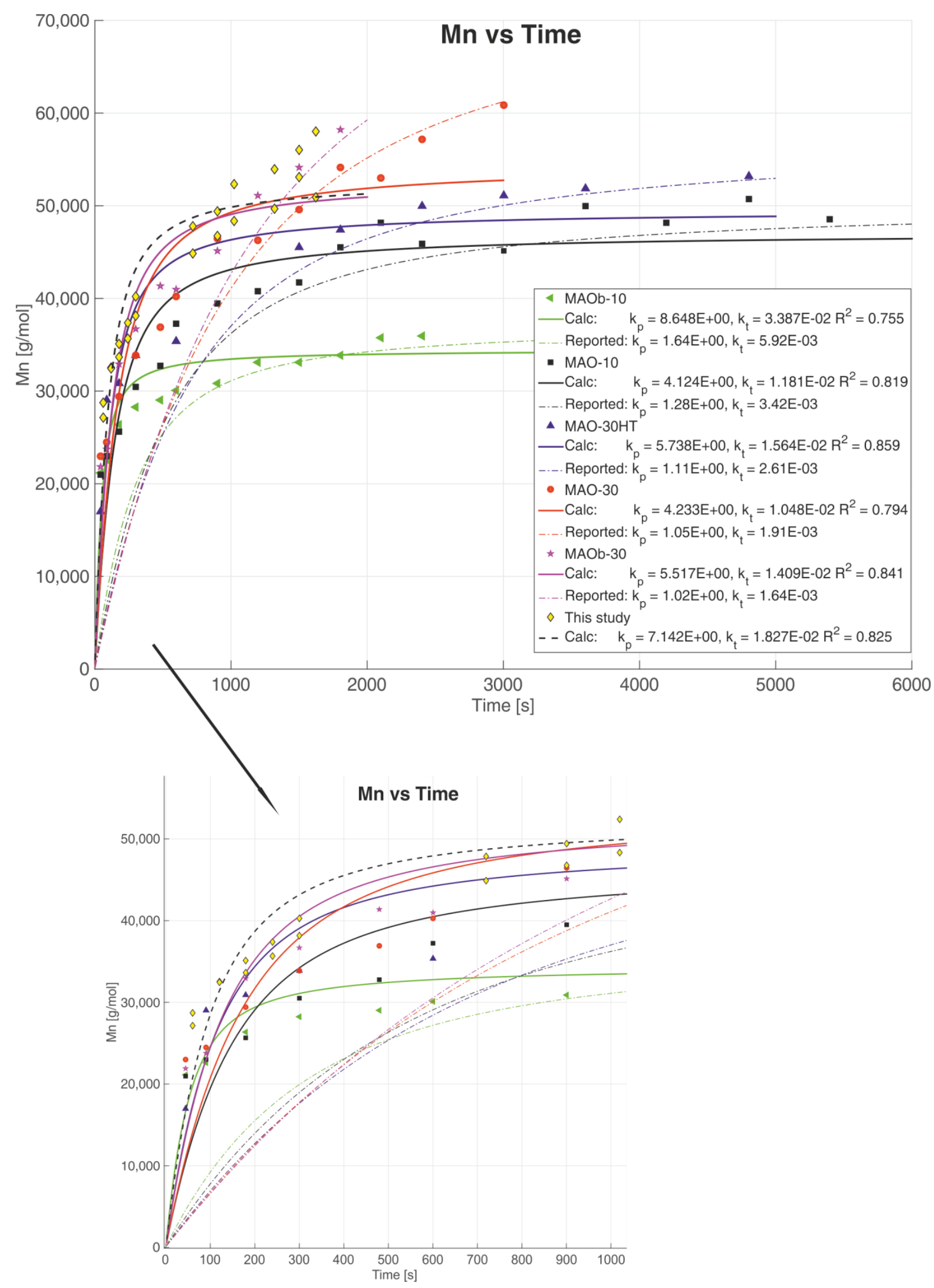

Figure S2. Reprocessed kinetic plots for the $\{\mathbf{S B I}\}-1 / \mathrm{MAO}$ system from ref. 12d: polymer yield (g) as function of time (s). The bold curves correspond to the non-linear regression calculated for the data points obtained at $<20 \%$ monomer conversion (MAOb-10: 5000 s; MAO-10: 6000 
s; MAO-30HT: 5000s; MAO-30: 3000 s; MAOb-30: 2000 s); the colored dash-dotted curves correspond to data plotted using the reported values of $k_{\mathrm{p}}$ and $k_{\mathrm{t}}$; the yellow points as well as the dashed black curve correspond to the kinetic data obtained in this study for the $\{\mathbf{S B I}\}-1 / \mathrm{MAO}$ system under identical conditions. 

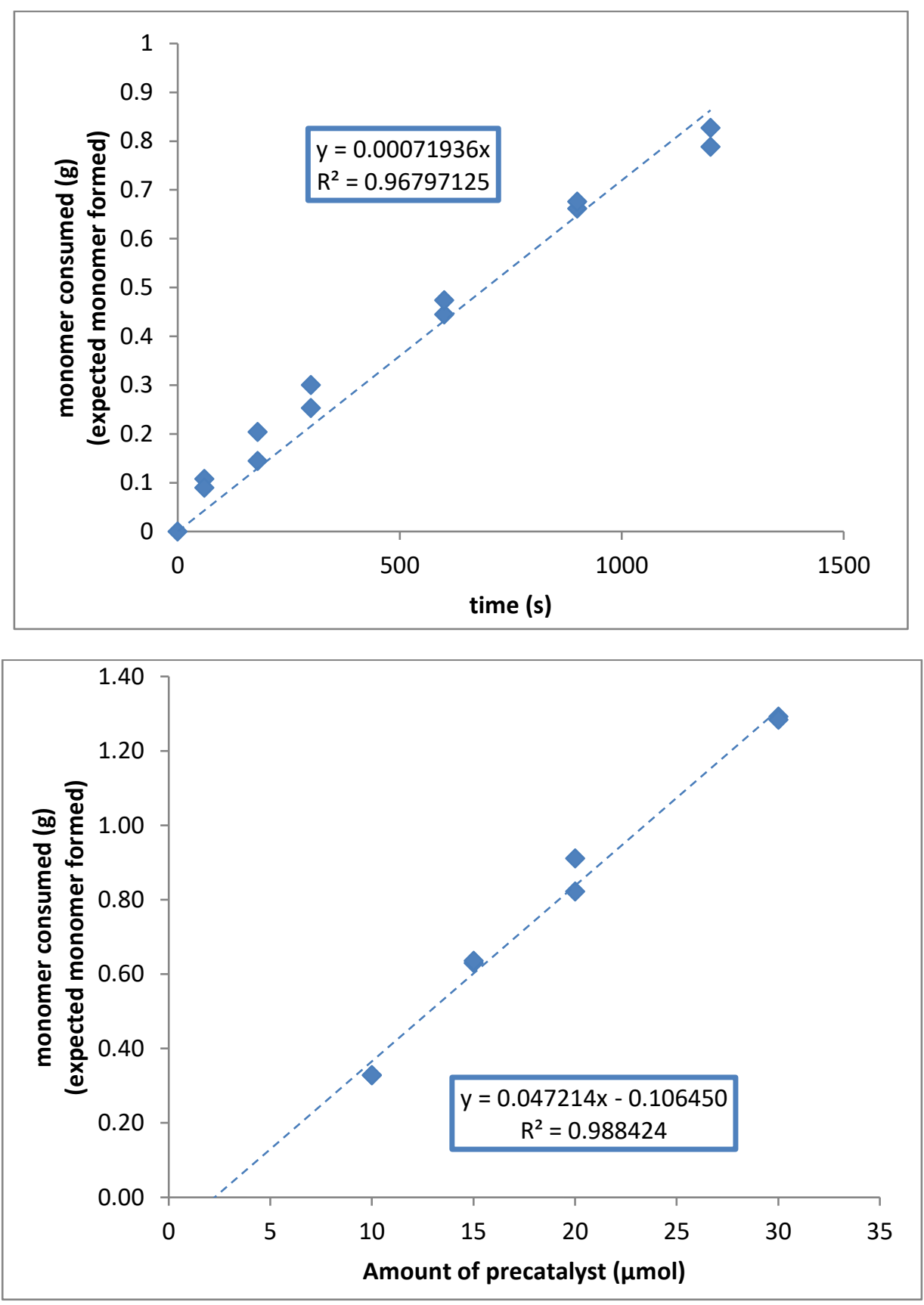


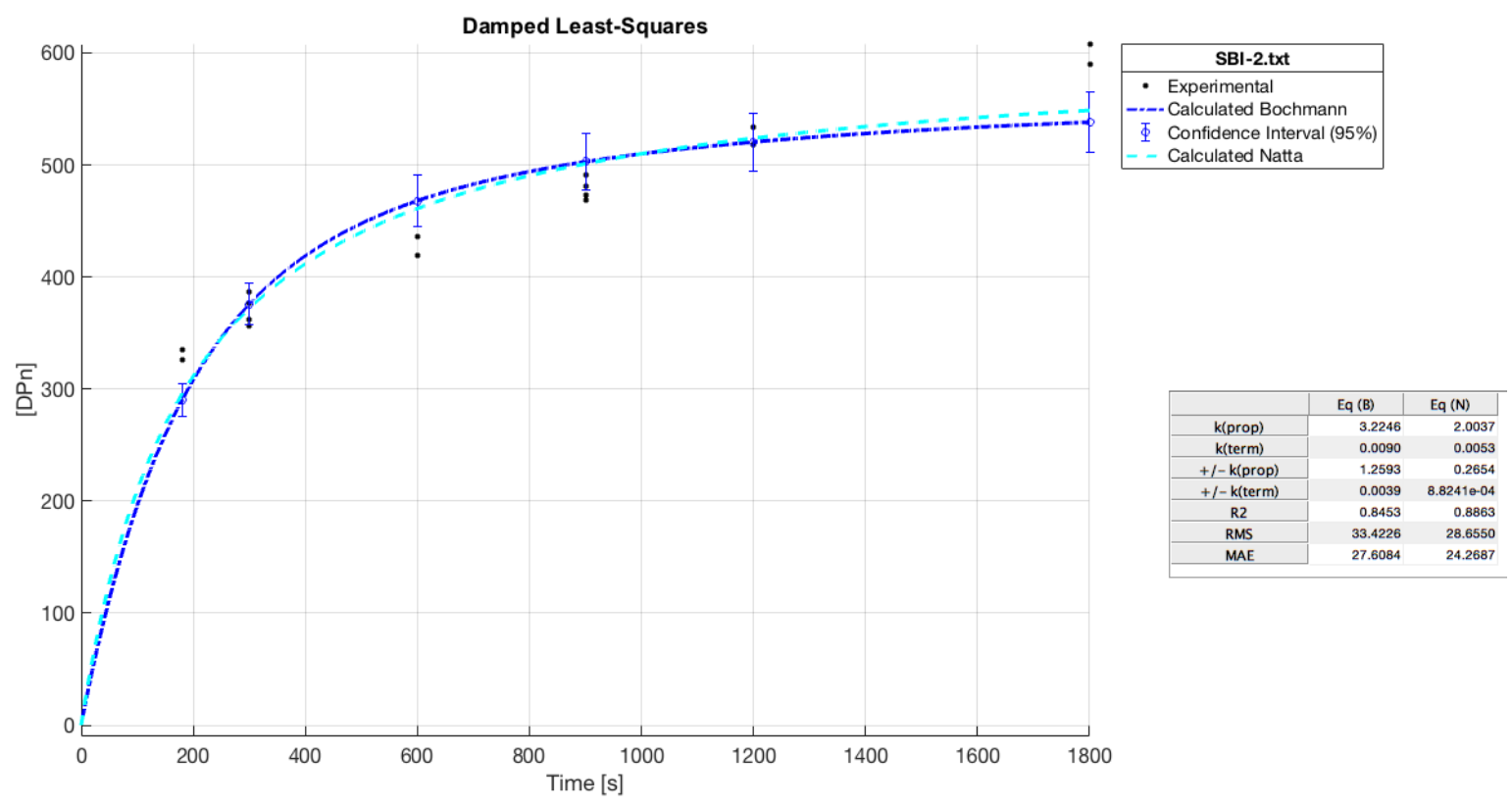

Figure S3. Kinetic plots for the $\{$ SBI $\}-2 / \mathrm{MAO}$ system (aging time $=60 \mathrm{~min} ; \mathrm{T}_{\text {aging }}=\mathrm{T}_{\mathrm{polym}}=$ $30{ }^{\circ} \mathrm{C}$ ). Polymer yield (g) as function of time (s) (top); polymer yield (g) as function of amount of precatalyst (middle); evolution of polymerization degree $\left(\mathrm{Dp}_{\mathrm{n}}\right)$ as function of time (s) (bottom): $\mathrm{R}_{(\text {Bochmann })}=0.845, \mathrm{RMS}_{(\mathrm{B})}=3.342 \cdot 10^{1}, \mathrm{R}^{2}$ (Natta) $=0.880, \mathrm{RMS}_{(\mathrm{N})}=2.865 \cdot 10^{1}, \mathrm{t}_{\mathrm{polym}}=$ $30 \mathrm{~min}$. 

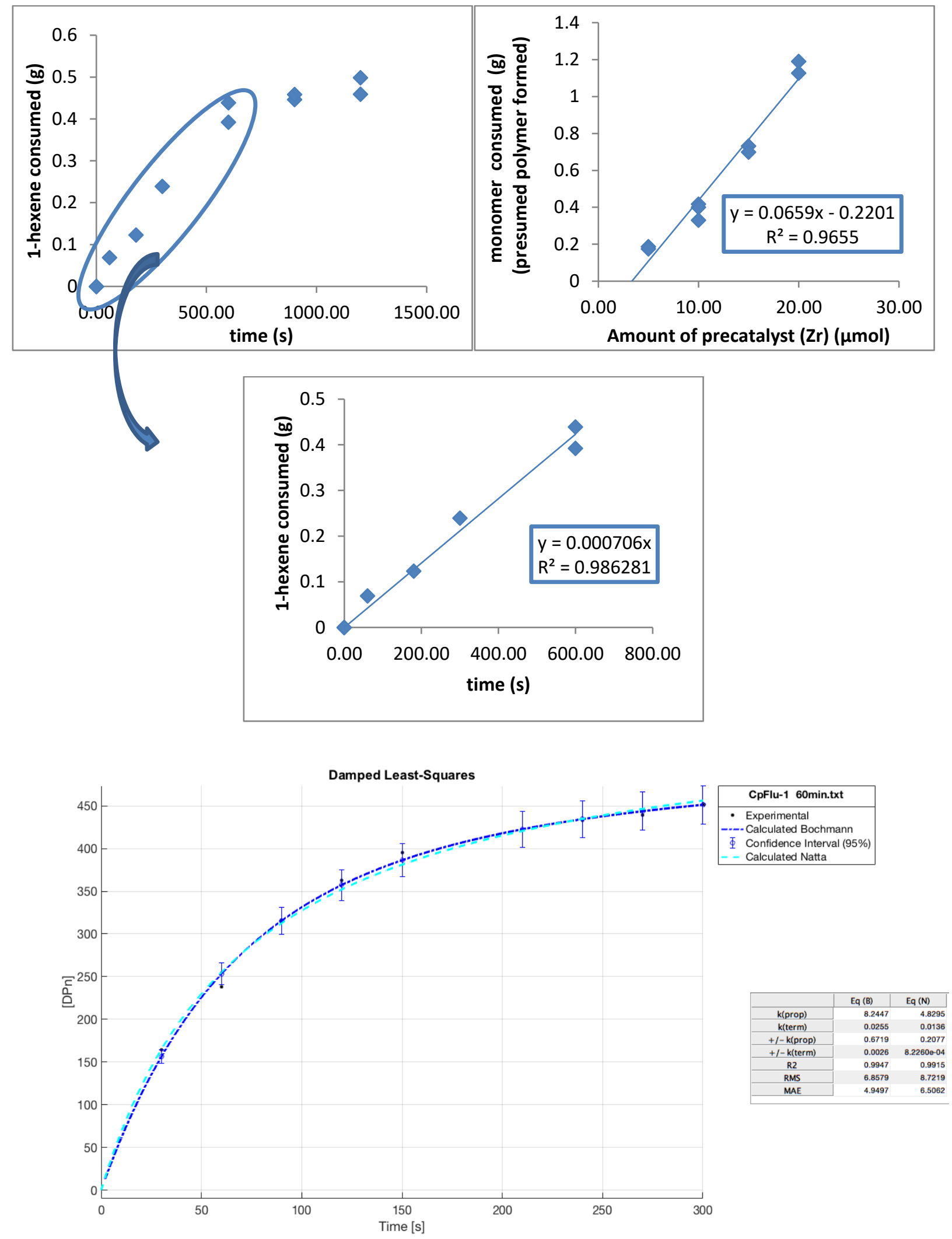

Figure S4. Polymer yield (g) as function of time (s) for the $\{\mathbf{C p} / \mathbf{F l u}\}-1 / \mathrm{MAO}$ system (aging time $\left.=60 \mathrm{~min} ; \mathrm{T}_{\text {aging }}=\mathrm{T}_{\text {polym }}=30{ }^{\circ} \mathrm{C}\right) . \quad \mathrm{R}_{(\text {Bochmann })}^{2}=0.923, \mathrm{RMS}_{(\mathrm{B})}=1.345 \cdot 10{ }^{1}, \mathrm{R}^{2}$ (Natta) $=$ $0.899, \mathrm{RMS}_{(\mathrm{N})}=1.536 \cdot 10^{1} ; \mathrm{t}_{\mathrm{polym}}=5 \mathrm{~min}($ top right $)$. 

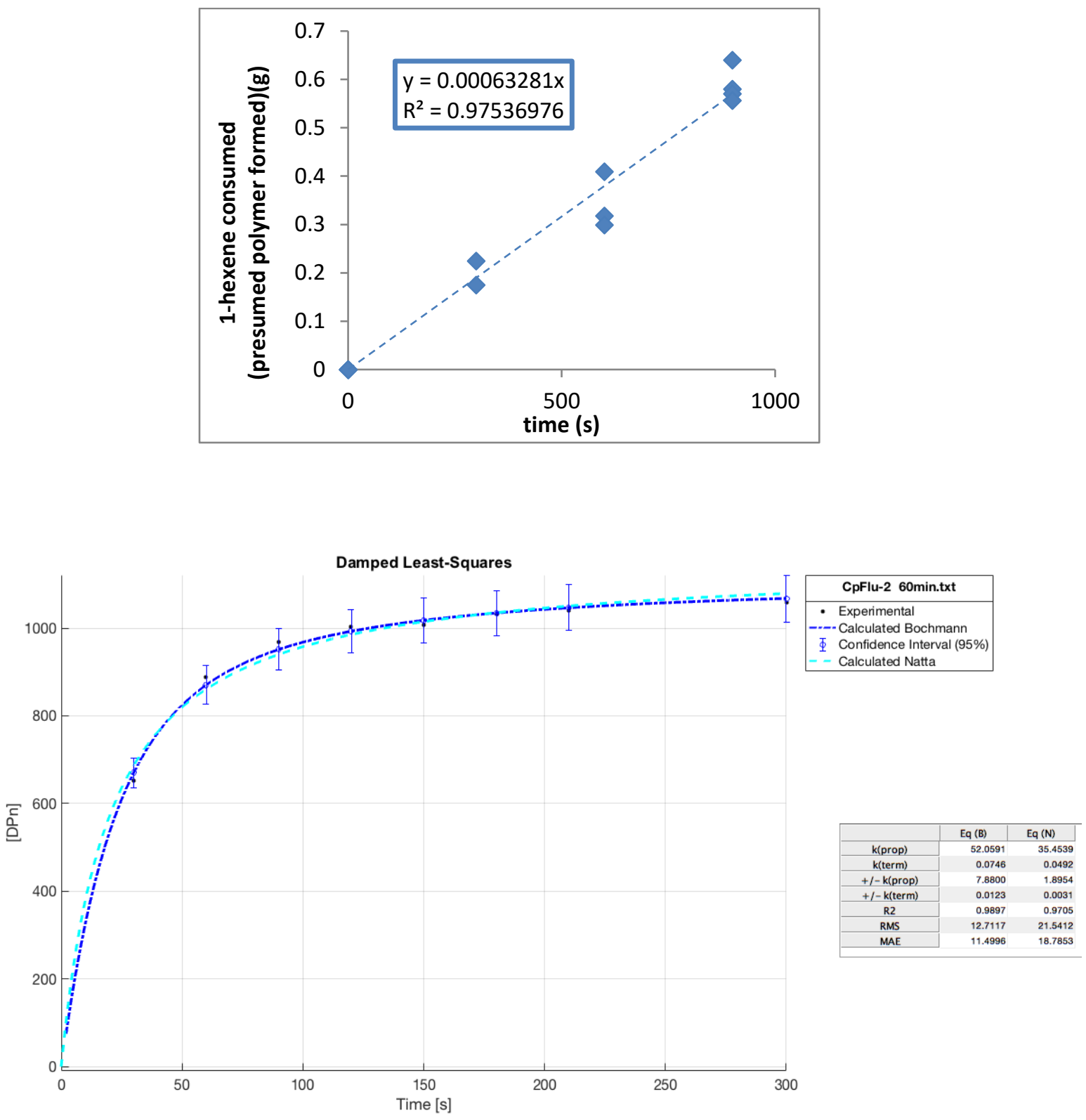

Figure S5. Kinetic plots for the $\{\mathbf{C p} / \mathbf{F l u}\}-2 / \mathrm{MAO}$ system $\left(\right.$ aging time $=60 \mathrm{~min} ; \mathrm{T}_{\text {aging }}=\mathrm{T}_{\mathrm{polym}}=$ $30{ }^{\circ} \mathrm{C}$ ). Polymer yield (g) as function of time (s) (top) and evolution of polymerization degree as function of time (bottom) $\mathrm{R}_{(\text {Bochmann })}=0.989, \mathrm{RMS}_{(\mathrm{B})}=1.271 \cdot 10^{1}, \mathrm{R}^{2}$ (Natta) $=0.971, \mathrm{RMS}_{(\mathrm{N})}=$ $2.154 \cdot 10^{1}$. 

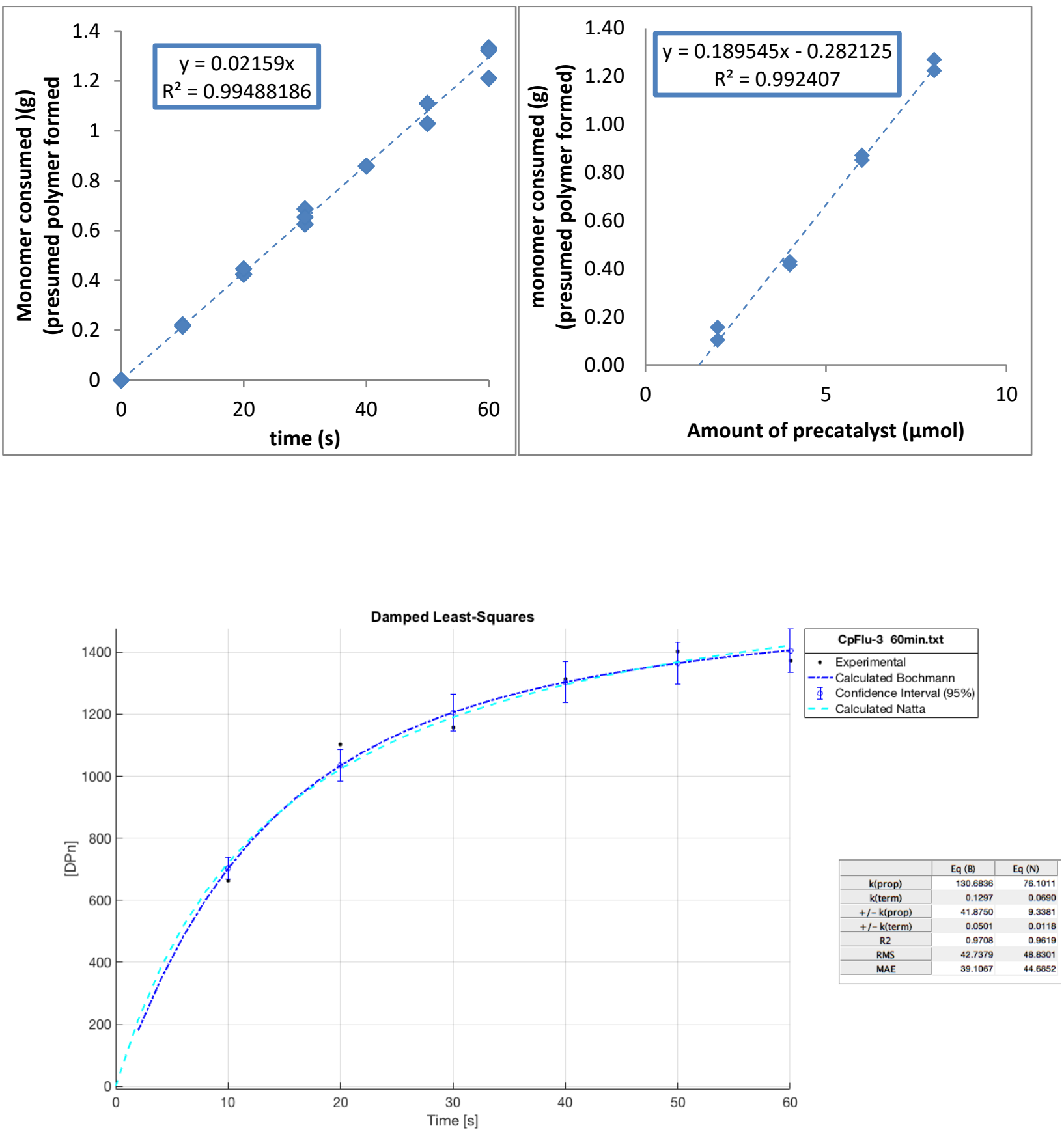

Figure S6. Kinetic plots for the $\{\mathbf{C p} /$ Flu $\}-3 / \mathrm{MAO}$ system (aging time $=60 \mathrm{~min} ; \mathrm{T}_{\text {aging }}=\mathrm{T}_{\mathrm{polym}}=$ $30{ }^{\circ} \mathrm{C}$ ). Polymer yield (g) as function of time (s) (left), polymer yield as function of amount of precatalyst (right) and evolution of polymerization degree as function of time (bottom), $\mathrm{R}_{(\text {Bochmann })}^{2}=0.971, \mathrm{RMS}_{(\mathrm{B})}=4.271 \cdot 10^{1} ; \mathrm{R}^{2}{ }_{(\mathrm{Natta})}=0.962, \mathrm{RMS}_{(\mathrm{N})}=4.883 \cdot 10^{1}$. 


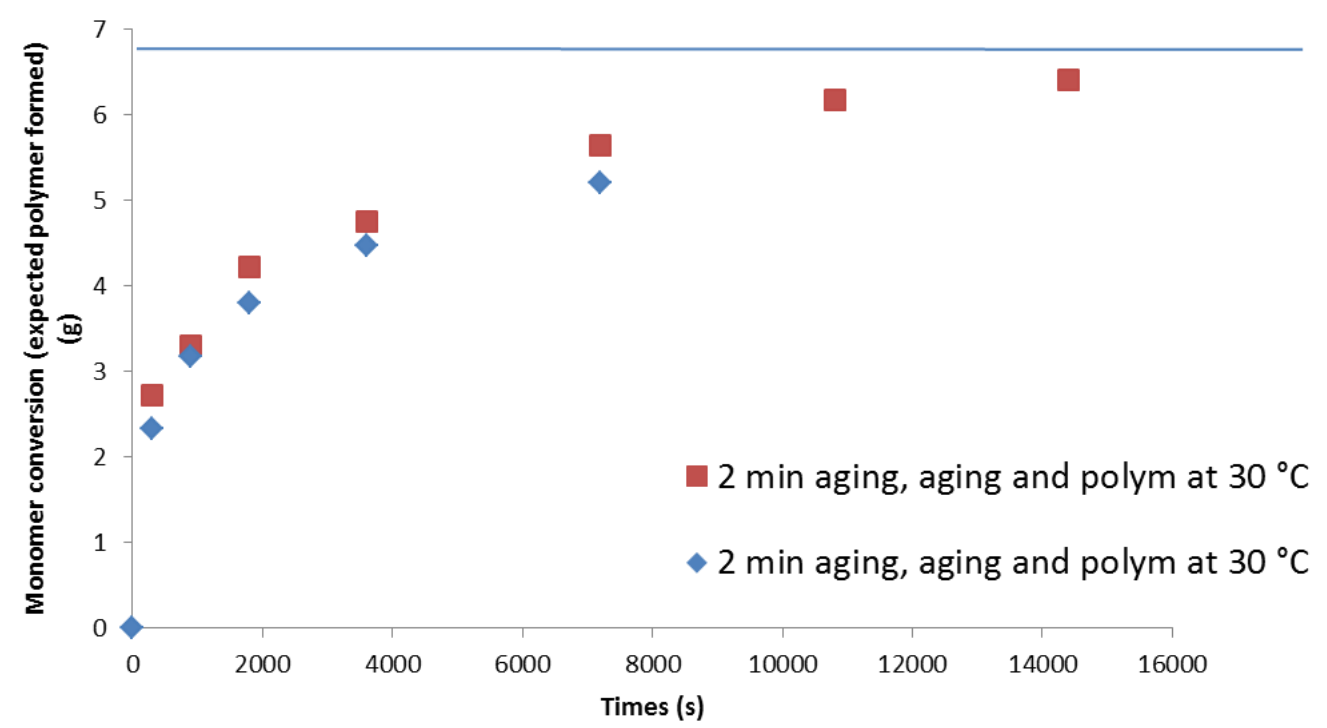

Figure S7. Kinetic plots obtained with the $\{\mathbf{C p} /$ Flu $\}-4 / \mathrm{MAO}$ system at $30{ }^{\circ} \mathrm{C}$ and $60{ }^{\circ} \mathrm{C}$. Conditions: $[\mathrm{Zr}]_{\mathrm{tot}}=0.20 \mathrm{mmol} \cdot \mathrm{L}^{-1},[\mathrm{MAO}] /[\mathrm{Zr}]_{\mathrm{tot}}=1000,[1 \text {-hexene }]_{0} /[\mathrm{Zr}]_{\text {tot }}=8060$, toluene. 

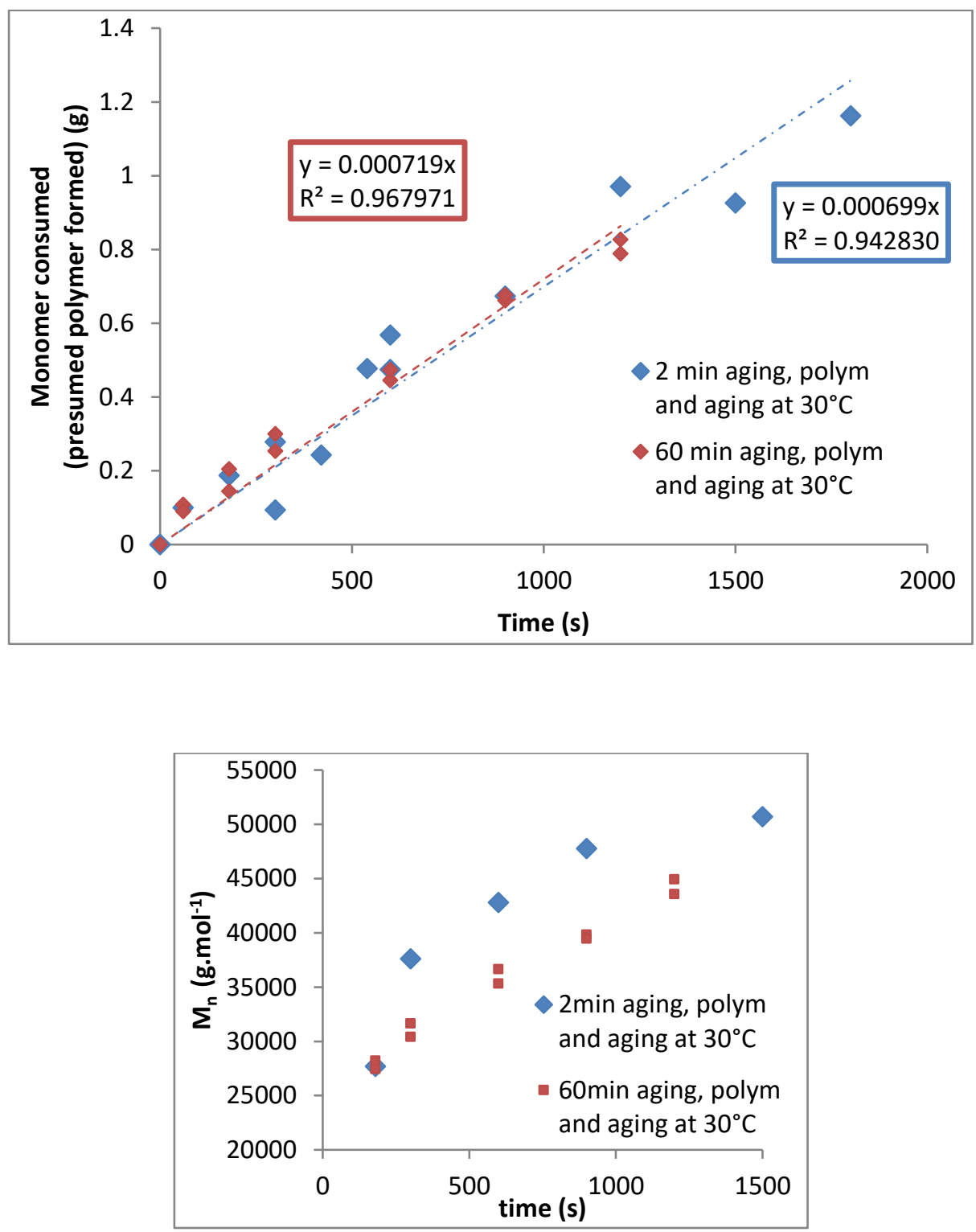


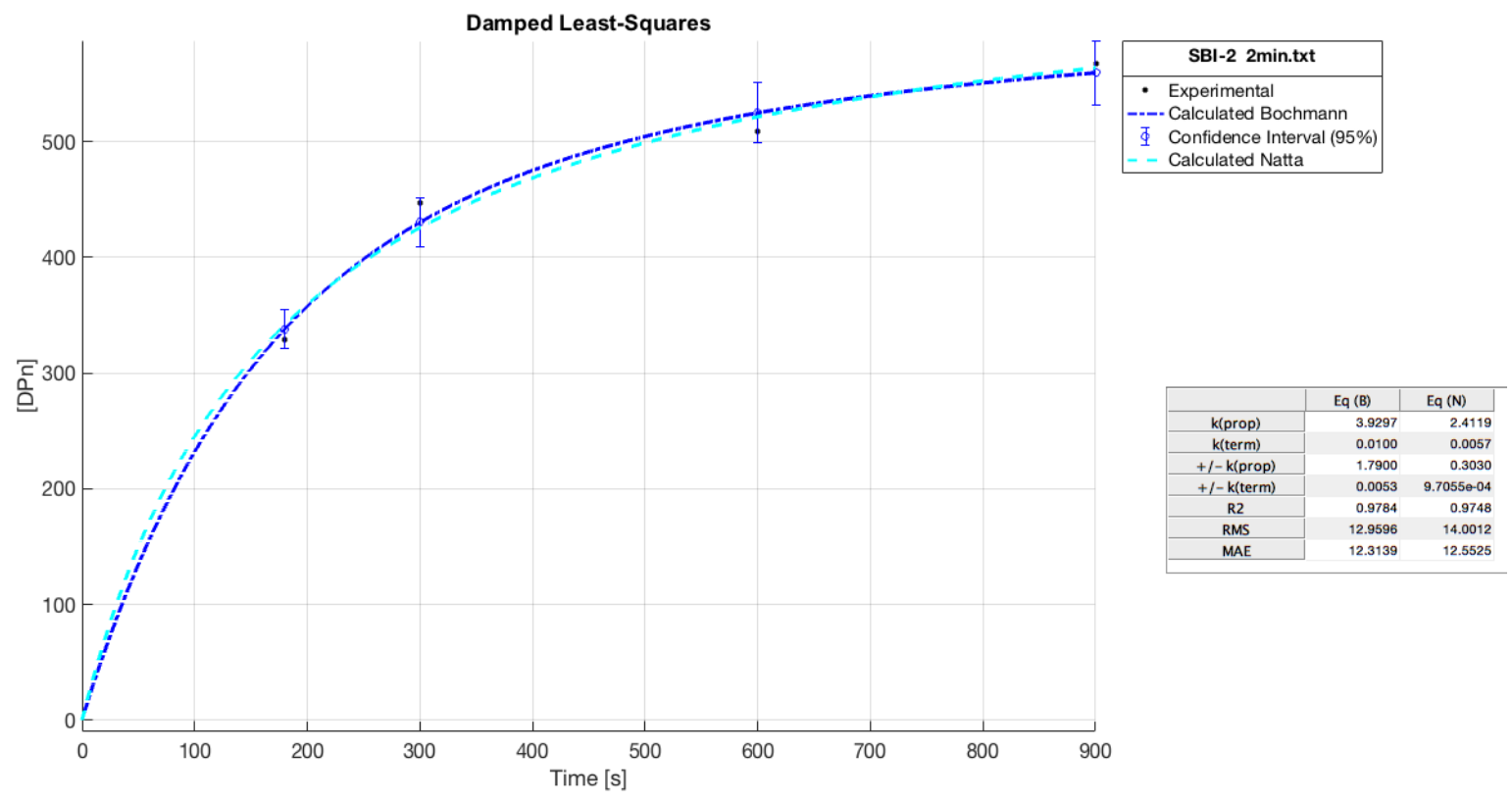

Figure S8. Kinetic plots obtained with the $\{$ SBI $\}-2 / M A O$ system at different aging times. Polymer yield (g) as function of time (s) (top) and polymerization degree as function of time(s) (Aging time $=2 \mathrm{~min}, \mathrm{~T}_{\text {aging }}=\mathrm{T}_{\mathrm{polym}}=30^{\circ} \mathrm{C}$ ) (middle) and evolution of polymerization degree as function of time (bottom); $\mathrm{R}^{2}$ (Bochmann) $=0.978, \mathrm{RMS}_{(\mathrm{B})}=1.296 \cdot 10^{1} ; \mathrm{R}^{2}{ }_{\text {(Natta) }}=0.974, \mathrm{RMS}_{(\mathrm{N})}=$ $1.400 \cdot 10^{1}$. Conditions: $[\mathrm{Zr}]_{\mathrm{tot}}=0.20 \mathrm{mmol} \cdot \mathrm{L}^{-1},[\mathrm{MAO}] /[\mathrm{Zr}]_{\mathrm{tot}}=1000,[1-\text { hexene }]_{0} /[\mathrm{Zr}]_{\mathrm{tot}}=$ 8060 , toluene, $\mathrm{T}_{\text {polym }}=30^{\circ} \mathrm{C}$.

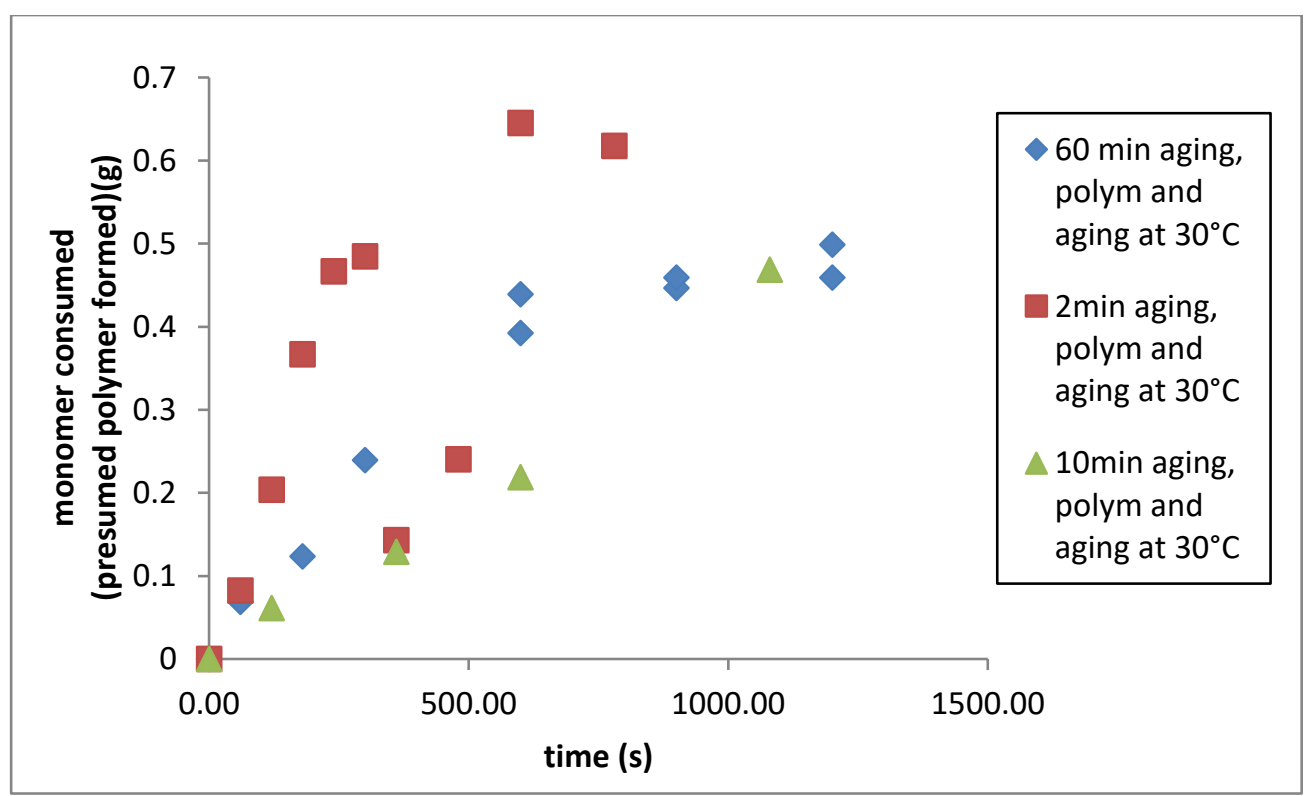



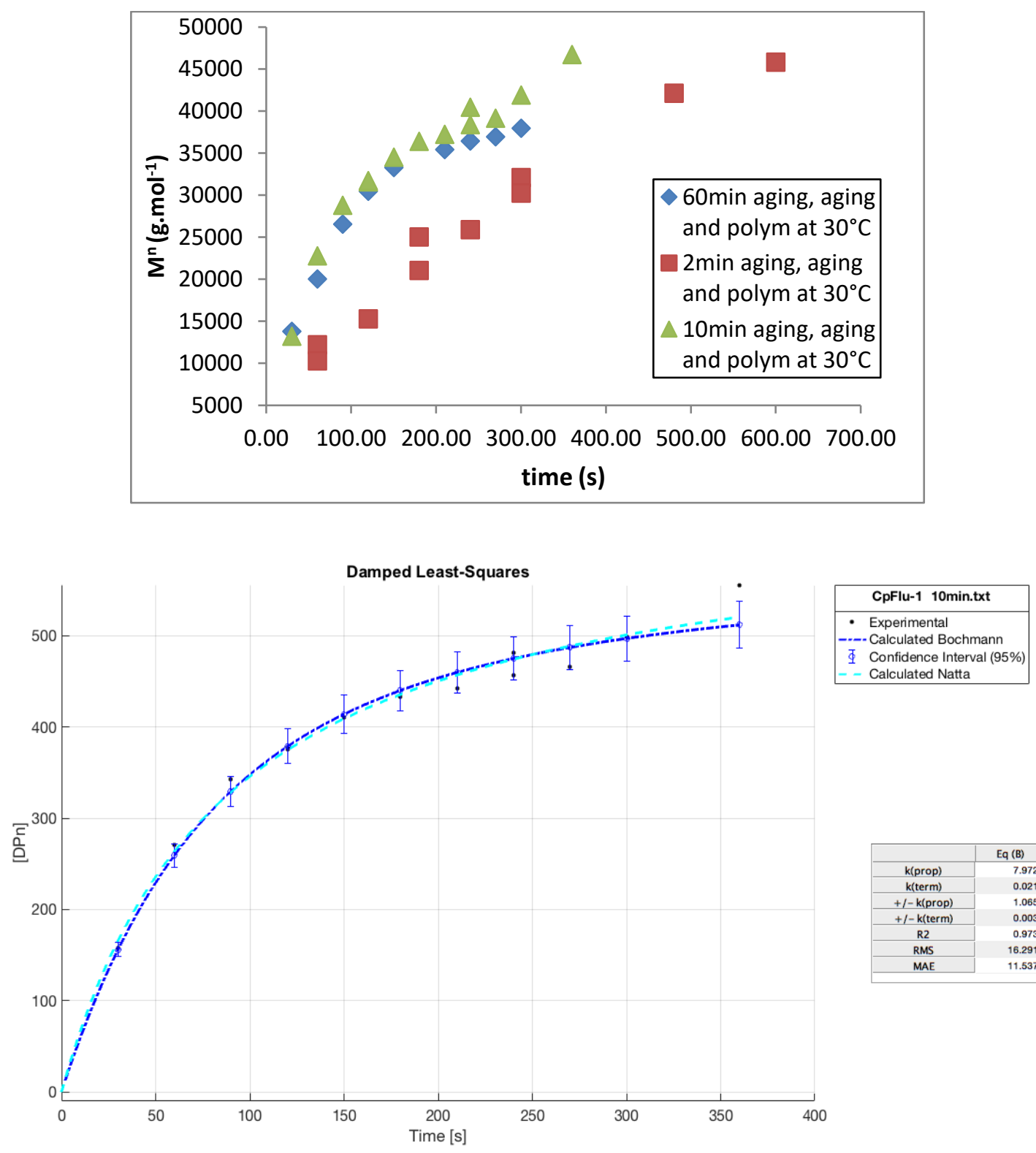

Calculated Natta

\begin{tabular}{|c|r|r|}
\hline & Eq (B) & \multicolumn{1}{|c|}{ Eq (N) } \\
\hline k(prop) & 7.9723 & 4.6440 \\
\hline k(term) & 0.0218 & 0.0115 \\
\hline +/-k(prop) & 1.0657 & 0.3580 \\
\hline +/-k(term) & 0.0037 & 0.0013 \\
\cline { 1 - 1 } R2 & 0.9736 & 0.9794 \\
\cline { 1 - 1 } RMS & 16.2915 & 14.3785 \\
\cline { 1 - 1 } MAE & 11.5371 & 10.4925 \\
\hline
\end{tabular}

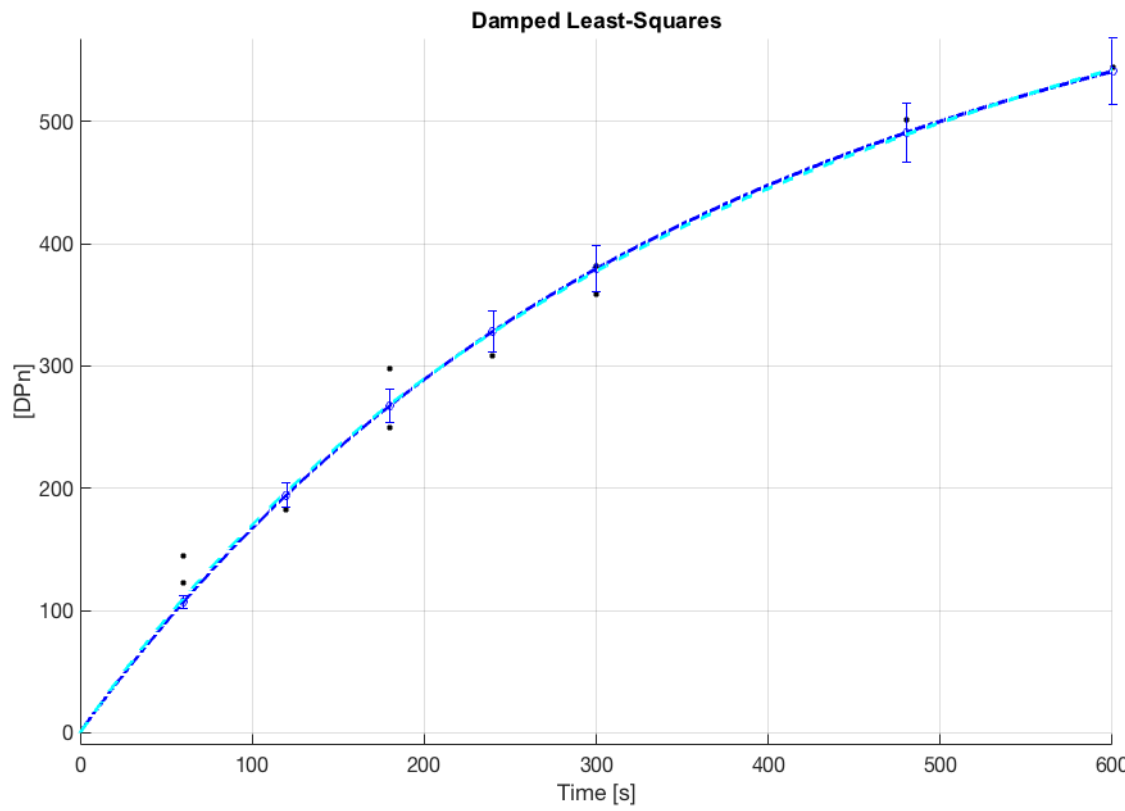

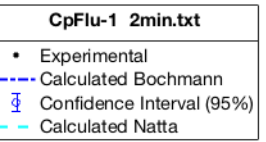

\begin{tabular}{|c|r|r|}
\hline & Eq (B) & \multicolumn{1}{|c|}{ Eq (N) } \\
\hline k(prop) & 2.4293 & 1.2930 \\
\cline { 1 - 1 } k(term) & 0.0049 & 0.0021 \\
\hline +l-k(prop) & 0.3503 & 0.1033 \\
\cline { 1 - 1 }+ /-k(term) & 0.0013 & $3.85449-04$ \\
\cline { 1 - 1 } R2 & 0.9778 & 0.9797 \\
\cline { 1 - 1 } RMS & 20.0773 & 19.2154 \\
\cline { 1 - 1 } RAE & 17.0666 & 16.6496 \\
\hline
\end{tabular}


Figure S9. Kinetic plots obtained with the $\{\mathbf{C p} / \mathbf{F l u}\}-1 / \mathrm{MAO}$ system with variation of the aging time. Polymer yield ( $\mathrm{g}$ ) as function of time (s) (top), Molecular weight $\left(\mathrm{M}_{\mathrm{n}}\right)$ as function of time (s) (middle), Polymerization degree as function of time (aging time $=2 \mathrm{~min}, \mathrm{~T}_{\text {aging }}=\mathrm{T}_{\text {polym }}=$ $\left.30{ }^{\circ} \mathrm{C}\right)($ bottom $($ down $)), \mathrm{R}_{(\text {Bochmann })}^{2}=0.977, \mathrm{RMS}_{(\mathrm{B})}=2.008 \cdot 10^{1} ; \mathrm{R}^{2}$ (Natta) $=0.979, \mathrm{RMS}_{(\mathrm{N})}$ $=1.922 \cdot 10^{1}$, (aging time $=10 \mathrm{~min}, \mathrm{~T}_{\text {aging }}=\mathrm{T}_{\text {polym }}=30^{\circ} \mathrm{C}$ ) (bottom (up)), $\mathrm{R}^{2}$ (Bochmann) $=0.974$, $\mathrm{RMS}_{(\mathrm{B})}=1.629 \cdot 10^{1} ; \mathrm{R}^{2}$ (Natta) $=0.979, \mathrm{RMS}_{(\mathrm{N})}=1.438 \cdot 10^{1}$. Conditions: $[\mathrm{Zr}]_{\mathrm{tot}}=0.20 \mathrm{mmol} \cdot \mathrm{L}^{-1}$, $[\mathrm{MAO}] /[\mathrm{Zr}]_{\text {tot }}=1000,[1-\text { hexene }]_{0} /[\mathrm{Zr}]_{\text {tot }}=8060$, toluene.
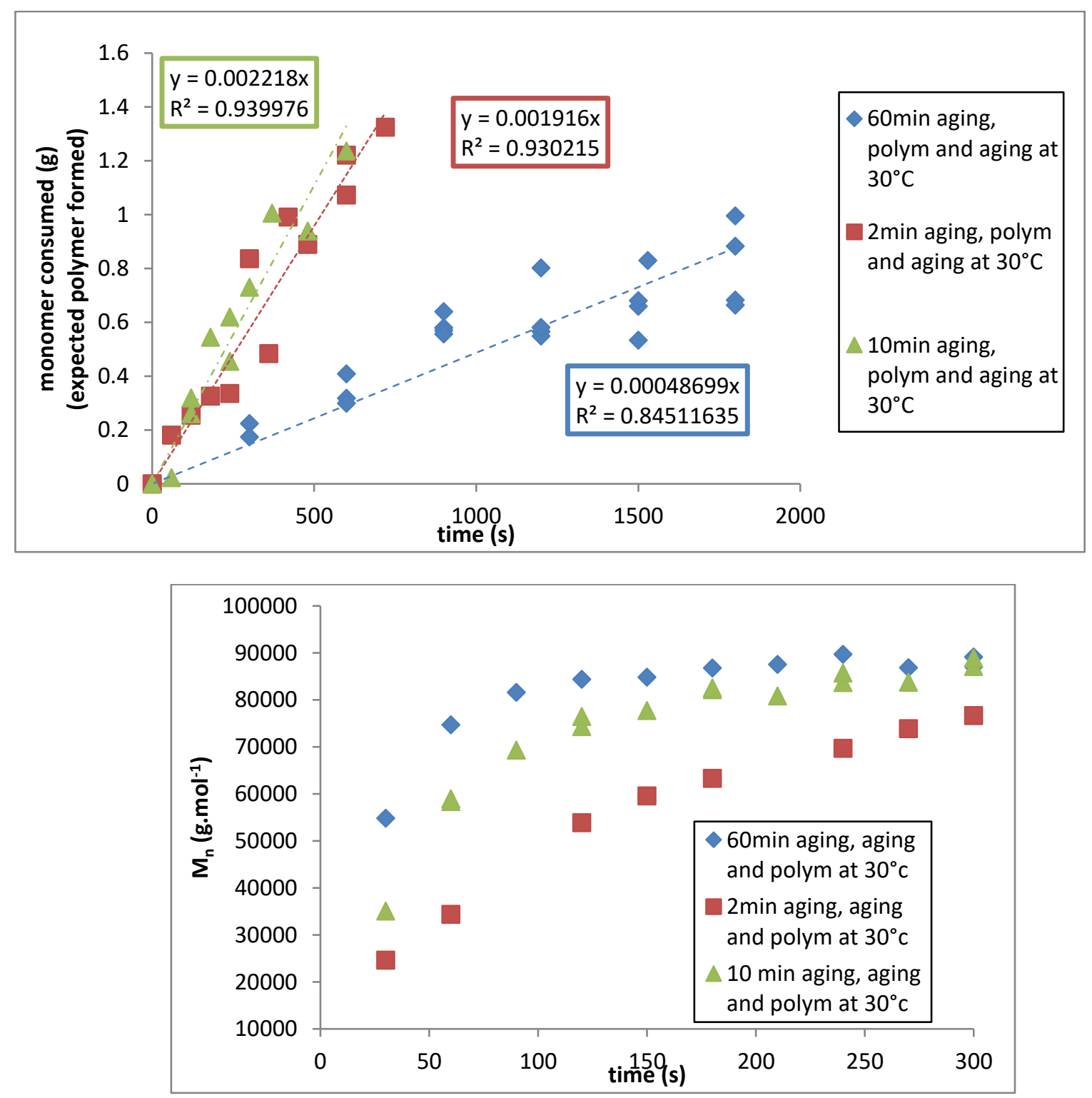

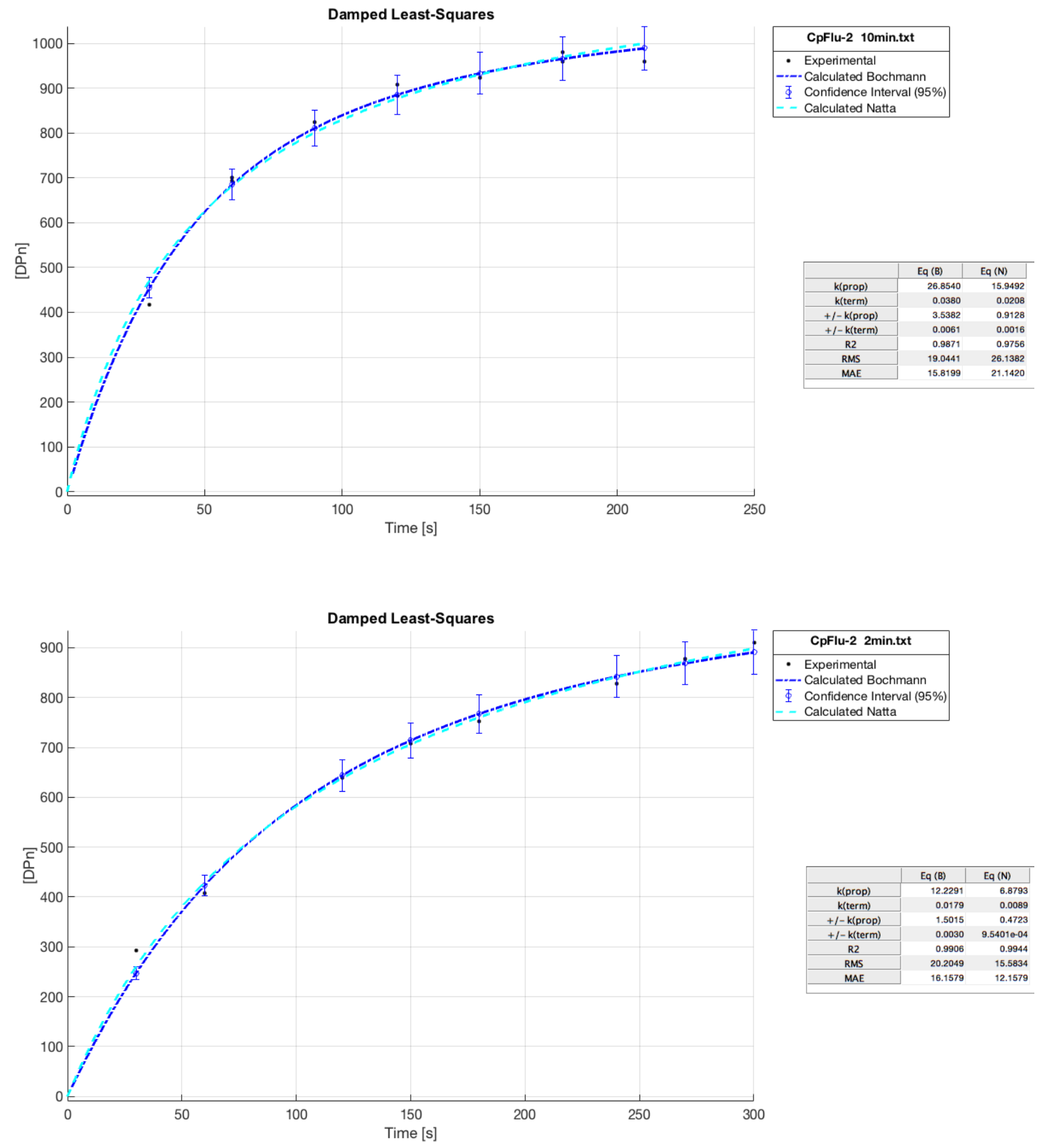

Figure S10. Kinetic plots obtained with the $\{\mathbf{C p} / \mathbf{F l u}\}-2 / \mathrm{MAO}$ system with variation of the aging time. Polymer yield (g) as function of time (s) (top), Molecular weight $\left(\mathrm{M}_{\mathrm{n}}\right)$ as function of time (s) (middle). Polymerization degree as function of time (aging time $=2 \mathrm{~min}, \mathrm{~T}_{\text {aging }}=\mathrm{T}_{\mathrm{polym}}=$ $\left.30{ }^{\circ} \mathrm{C}\right)$ (bottom (down)) $\mathrm{R}_{(\text {Bochmann })}=0.991, \mathrm{RMS}_{(\mathrm{B})}=2.020 \cdot 10^{1} ; \mathrm{R}^{2}{ }_{\text {(Natta) }}=0.994, \mathrm{RMS}_{(\mathrm{N})}$ $=1.558 \cdot 10^{1}$; (Aging time $\left.=10 \mathrm{~min}, \mathrm{~T}_{\text {aging }}=\mathrm{T}_{\text {polym }}=30^{\circ} \mathrm{C}\right)\left(\right.$ bottom (up)) $\mathrm{R}_{(\text {Bochmann })}^{2}=0.987$, $\mathrm{RMS}_{(\mathrm{B})}=1.90410^{1} ; \mathrm{R}^{2}$ (Natta) $=0.976, \mathrm{RMS}_{(\mathrm{N})}=1.595 \cdot 10^{1}$. Conditions: $[\mathrm{Zr}]_{\mathrm{tot}}=0.20 \mathrm{mmol} \cdot \mathrm{L}^{-1}$, $[\mathrm{MAO}] /[\mathrm{Zr}]_{\text {tot }}=1000,[1-\text { hexene }]_{0} /[\mathrm{Zr}]_{\text {tot }}=8060$, toluene. 

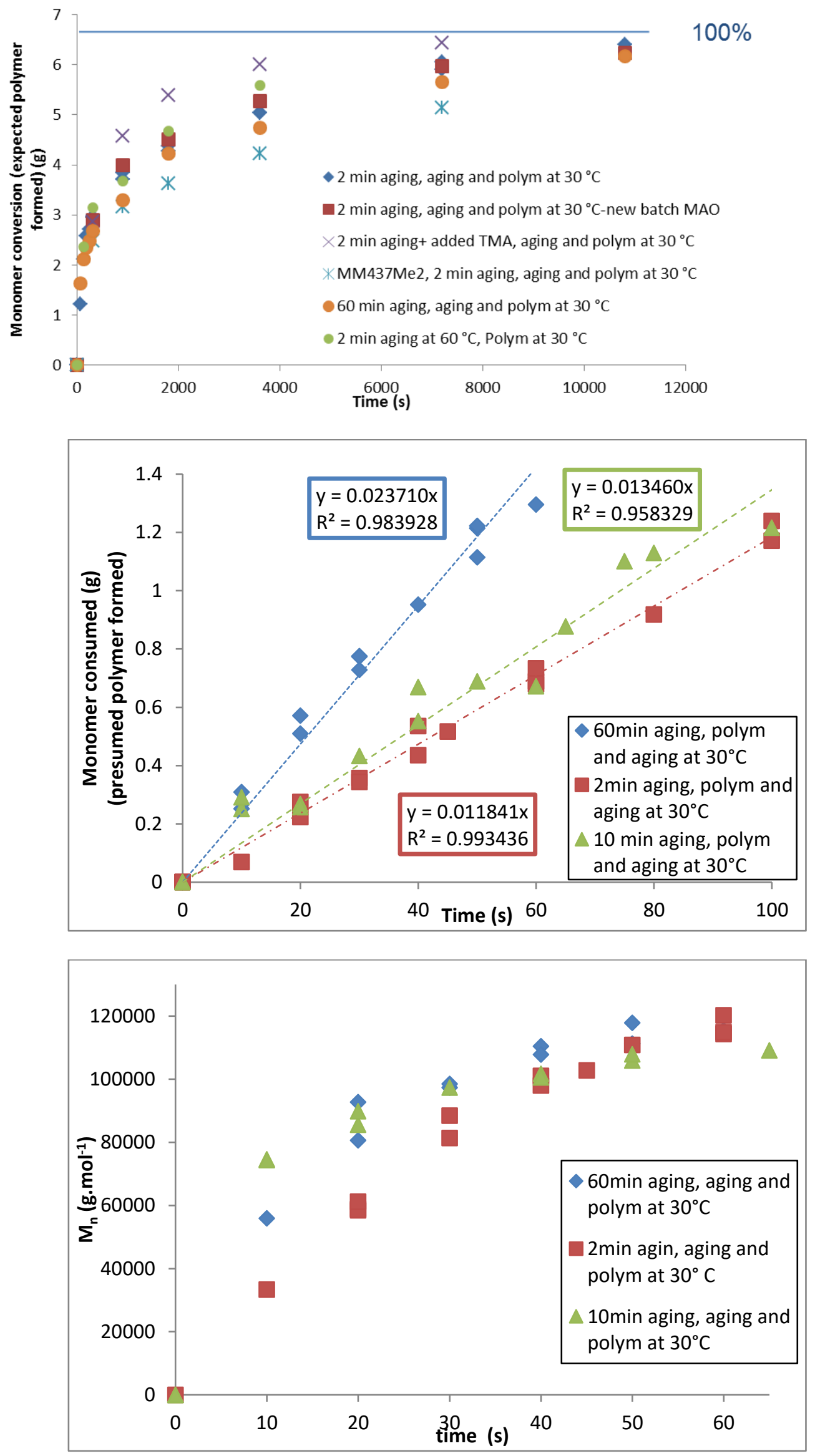

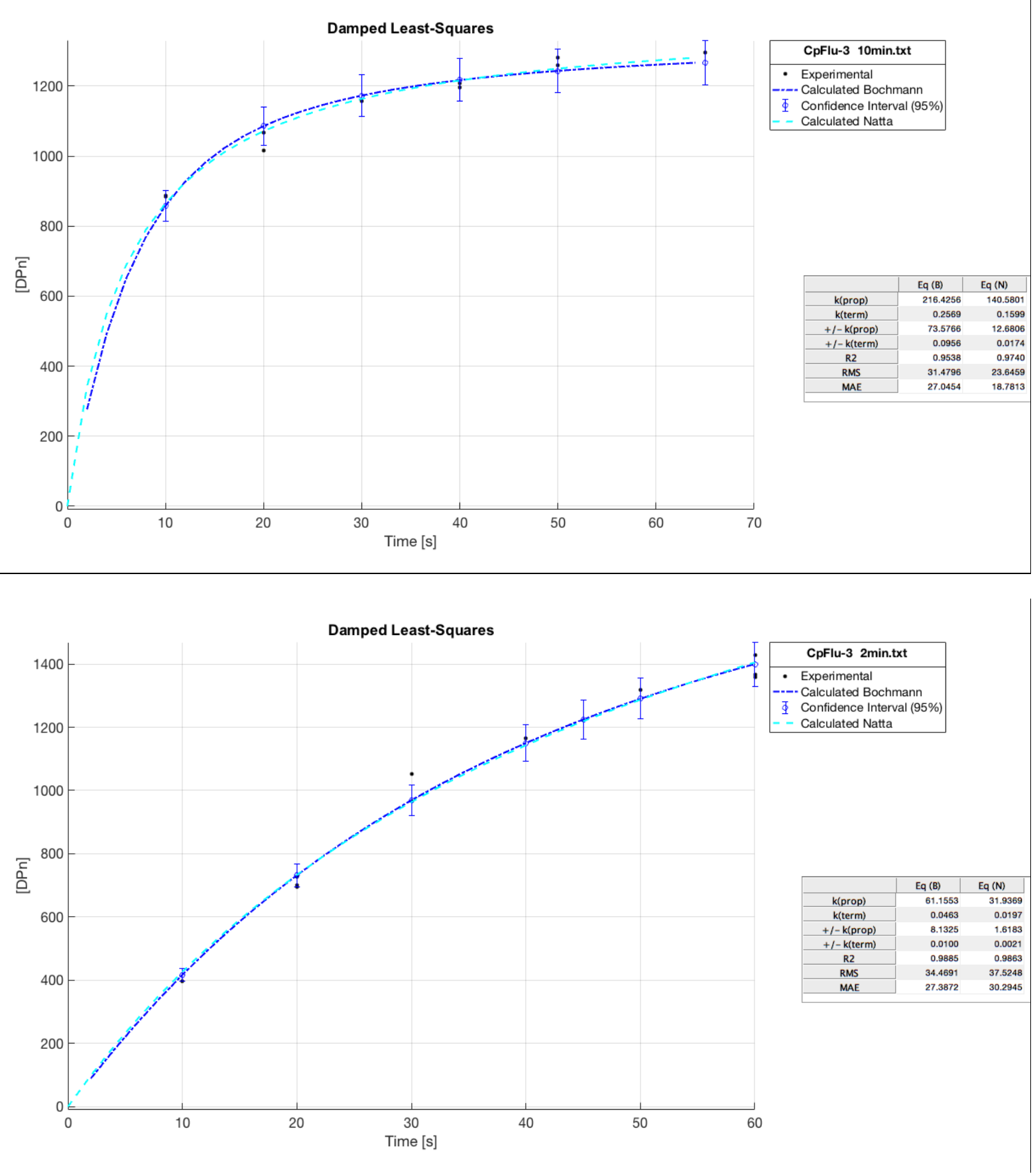

Figure S11. Kinetic plots obtained with the $\{\mathbf{C p} /$ Flu $\}-3 / \mathrm{MAO}$ system with variation of aging time. Polymer yield ( $\mathrm{g}$ ) as function of time (s) (top), Polymerization degree as function of time $\left(\right.$ aging time $\left.=2 \min , \mathrm{T}_{\text {aging }}=\mathrm{T}_{\text {polym }}=30{ }^{\circ} \mathrm{C}\right)($ bottom $($ down $)) \mathrm{R}_{(\text {Bochmann })}^{2}=0.989, \mathrm{RMS}_{(\mathrm{B})}=$ $3.447 \cdot 10^{1}, \mathrm{R}^{2}$ (Natta) $=0.986, \mathrm{RMS}_{(\mathrm{N})}=3.752 \cdot 10^{1} ;\left(\right.$ aging time $\left.=10 \mathrm{~min}, \mathrm{~T}_{\text {aging }}=\mathrm{T}_{\text {polym }}=30^{\circ} \mathrm{C}\right)$

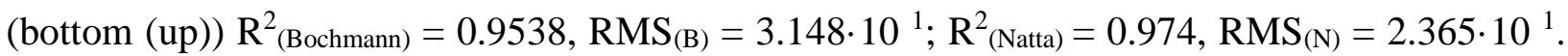
Conditions: $[\mathrm{Zr}]_{\mathrm{tot}}=0.20 \mathrm{mmol} \cdot \mathrm{L}^{-1},[\mathrm{MAO}] /[\mathrm{Zr}]_{\mathrm{tot}}=1000,[1-\text { hexene }]_{0} /[\mathrm{Zr}]_{\text {tot }}=8060$, toluene, $\mathrm{T}_{\text {polym }}=30{ }^{\circ} \mathrm{C}$. 


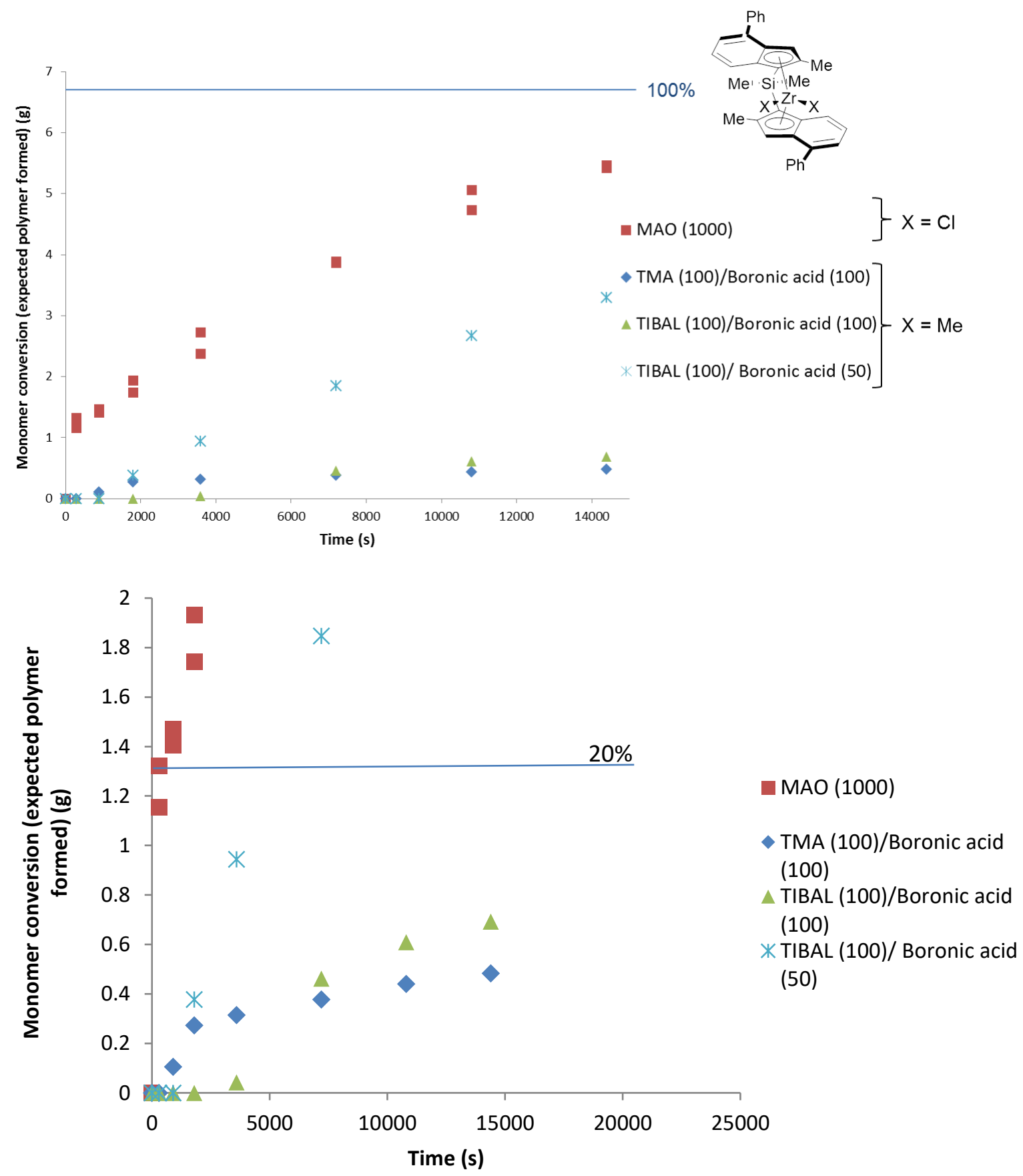

Figure S12. Kinetic plots obtained with $\{\mathbf{S B I}\}-2$ and $\{\mathbf{S B I}\}-2 \mathbf{a}$ using different coactivators. Conditions: $[\mathrm{Zr}]_{\mathrm{tot}}=0.20 \mathrm{mmol} \cdot \mathrm{L}^{-1},[1 \text {-hexene }]_{0} /[\mathrm{Zr}]_{\mathrm{tot}}=8060$, toluene, Aging time $=2 \mathrm{~min}$, $\mathrm{T}_{\text {aging }}=\mathrm{T}_{\text {polym }}=30^{\circ} \mathrm{C}$. 

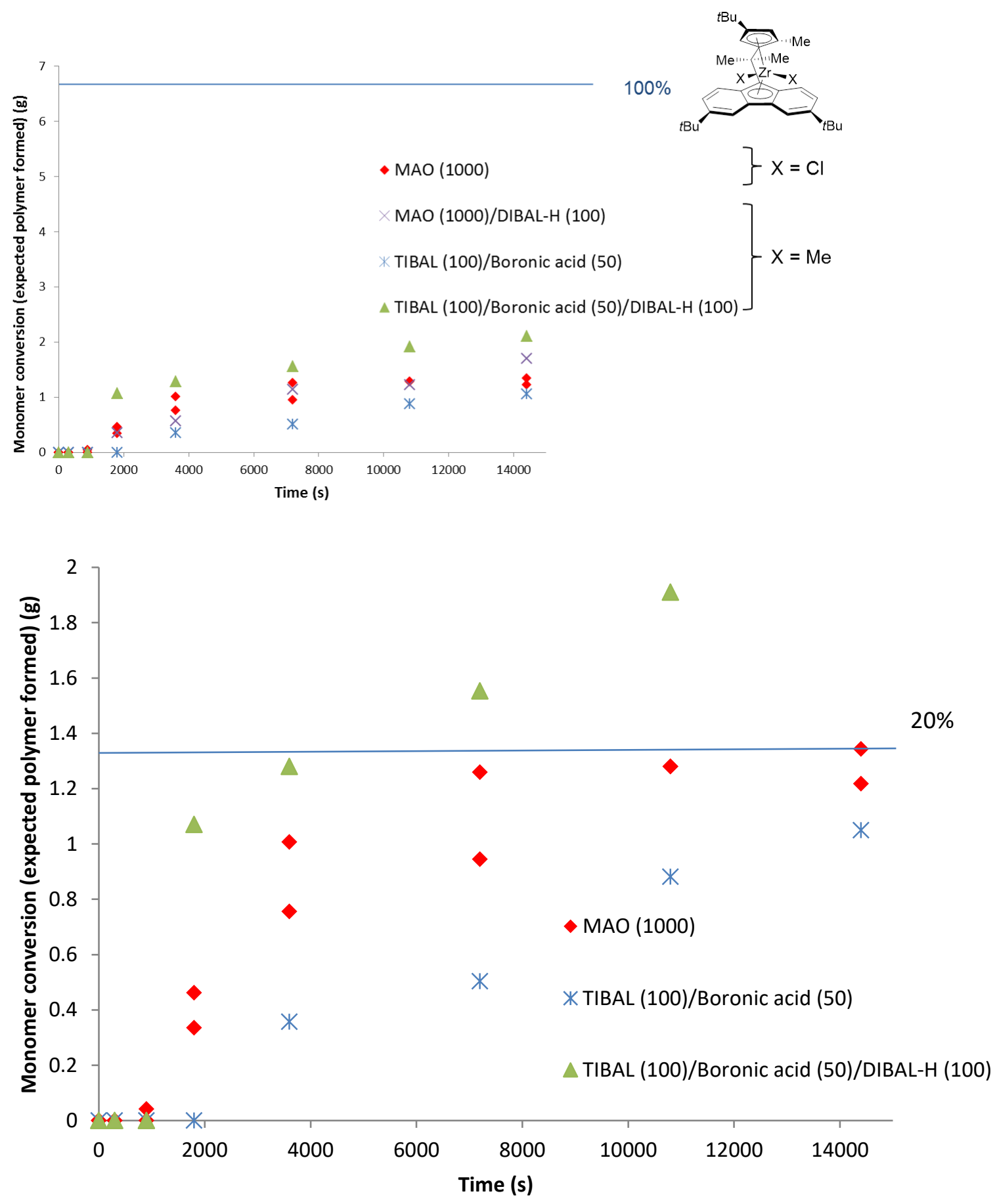

Figure S13. Kinetic plots obtained with $\{\mathbf{C p} / \mathbf{F l u}\}-1$ and $\{\mathbf{C p} / \mathbf{F l u}\}-1 a$ using different coactivators. Conditions: $[\mathrm{Zr}]_{\mathrm{tot}}=0.20 \mathrm{mmol} \cdot \mathrm{L}^{-1},[1-\text { hexene }]_{0} /[\mathrm{Zr}]_{\mathrm{tot}}=8060$, toluene, Aging time $=2 \mathrm{~min}, \mathrm{~T}_{\text {aging }}=\mathrm{T}_{\text {polym }}=30^{\circ} \mathrm{C}$. 

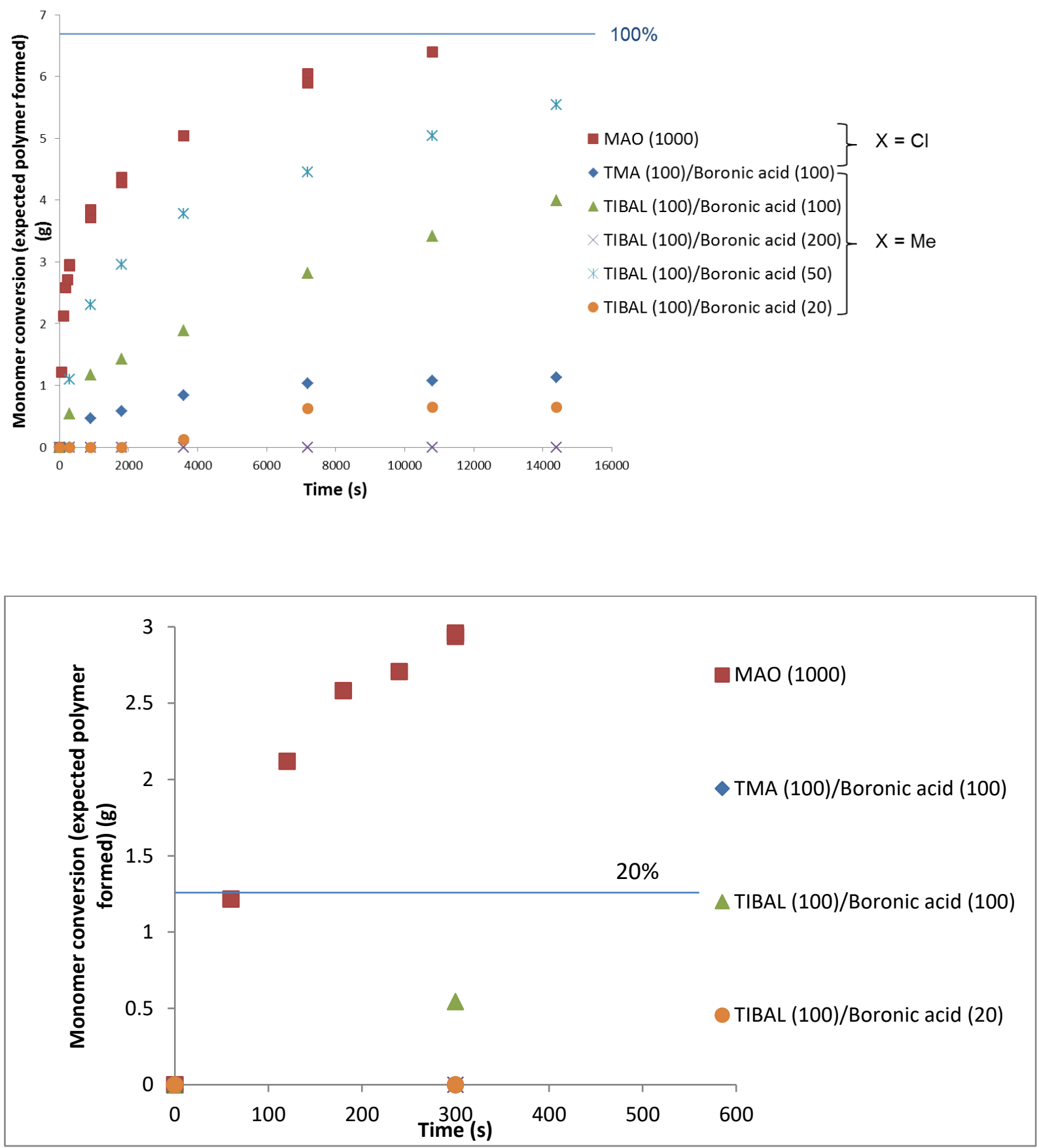

Figure S14. Kinetic plots obtained with $\{\mathbf{C p} / \mathbf{F l u}\}-3$ and $\{\mathbf{C p} / \mathbf{F l u}\}-3 a$ using different coactivators (top), and kinetic plot for the amount of produced polymer vs time for different TIBAL $/\left(\mathrm{C}_{6} \mathrm{~F}_{5}\right) \mathrm{B}(\mathrm{OH})_{2}$ ratios (bottom). Conditions: $[\mathrm{Zr}]_{\text {tot }}=0.20 \mathrm{mmol} \cdot \mathrm{L}^{-1},[\mathrm{MAO}] /[\mathrm{Zr}]_{\mathrm{tot}}=$ $1000,[1-\text { hexene }]_{0} /[\mathrm{Zr}]_{\text {tot }}=8060$, toluene, $\mathrm{T}_{\text {aging }}=\mathrm{T}_{\text {polym }}=30^{\circ} \mathrm{C}$. 


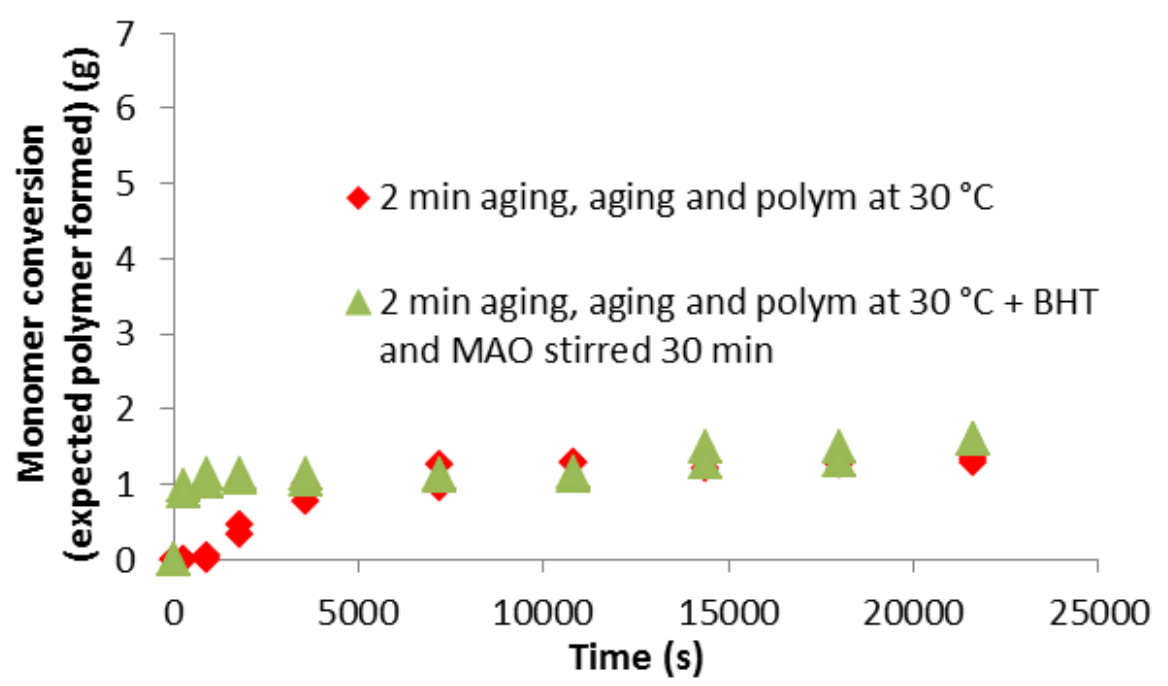

Figure S15. Kinetic plots obtained for $\{\mathbf{C p} / \mathbf{F l u}\}-\mathbf{1}$ activated with BHT-modified MAO (MAO stirred for $30 \mathrm{~min}$ with BHT, $[\mathrm{BHT}] /[\mathrm{MAO}]=0.5$ ). Conditions: $[\mathrm{Zr}]_{\mathrm{tot}}=0.20 \mathrm{mmol} \cdot \mathrm{L}^{-1}$, $[\mathrm{MAO}] /[\mathrm{Zr}]_{\mathrm{tot}}=1000,[1-\text { hexene }]_{0} /[\mathrm{Zr}]_{\mathrm{tot}}=8060$, toluene.
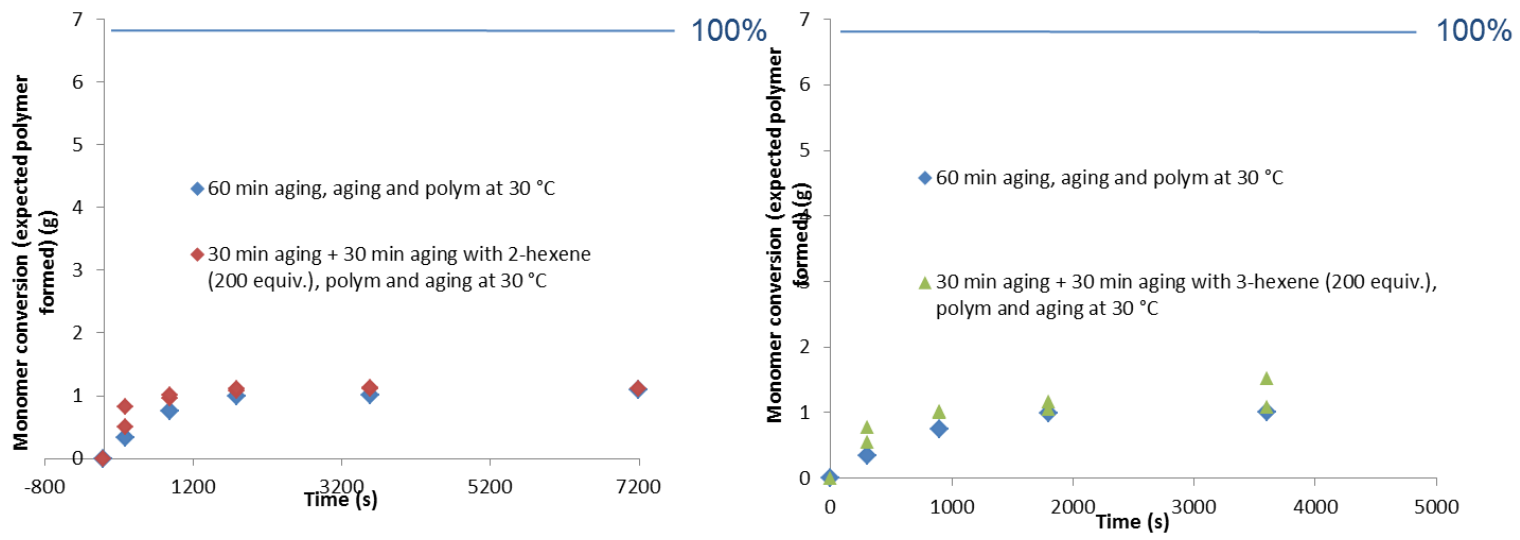

Figure S16. Kinetic plots obtained with the $\{\mathbf{C p} / \mathbf{F l u}\}-1 / \mathrm{MAO}$ system in the presence of 2hexene (left) and 3-hexene (right). Conditions: $[\mathrm{Zr}]_{\mathrm{tot}}=0.20 \mathrm{mmol} \cdot \mathrm{L}^{-1},[\mathrm{MAO}] /[\mathrm{Zr}]_{\mathrm{tot}}=1000$, $[\text { hexene }]_{0} /[\mathrm{Zr}]_{\text {tot }}=8060$, toluene. 


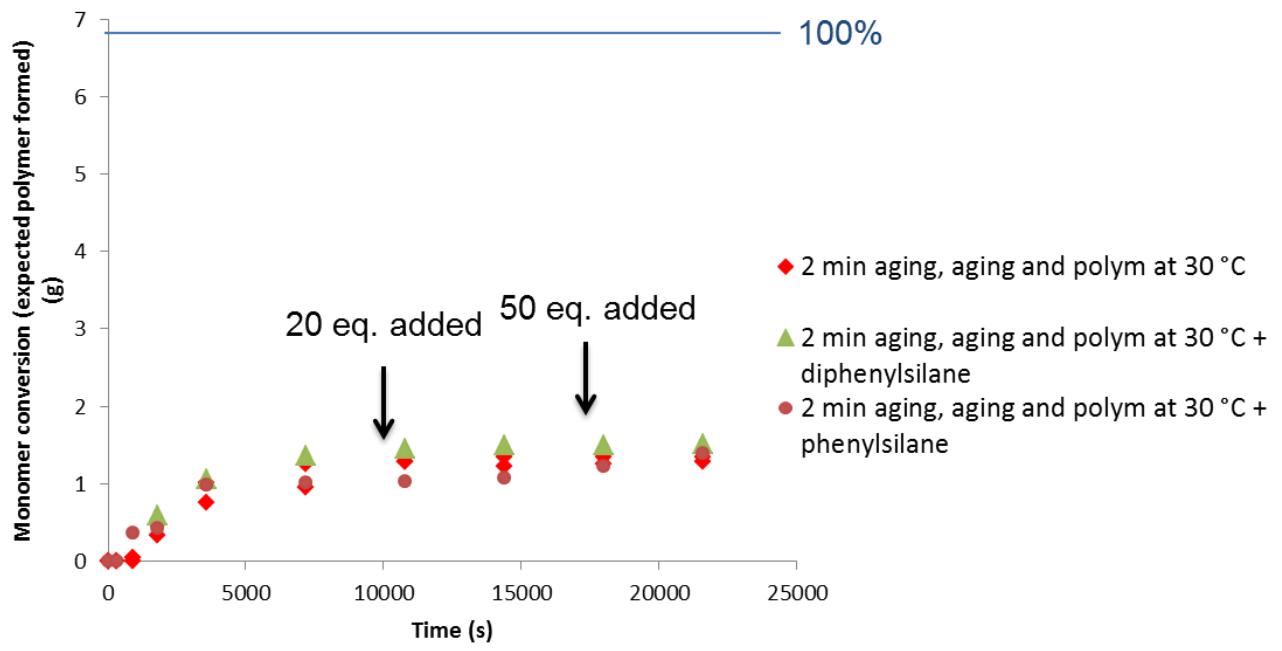

Figure S17. Kinetic plots obtained with the $\{\mathbf{C p} / \mathbf{F l u}\}-1 / \mathrm{MAO}$ system in the presence of $\mathrm{Ph}_{2} \mathrm{SiH}_{2}$ or $\mathrm{PhSiH}_{3}$. Conditions: $[\mathrm{Zr}]_{\text {tot }}=0.20 \mathrm{mmol} \cdot \mathrm{L}^{-1},[\mathrm{MAO}] /[\mathrm{Zr}]_{\text {tot }}=1000,[1-$ hexene $]_{0} /[\mathrm{Zr}]_{\text {tot }}=8060$, toluene.
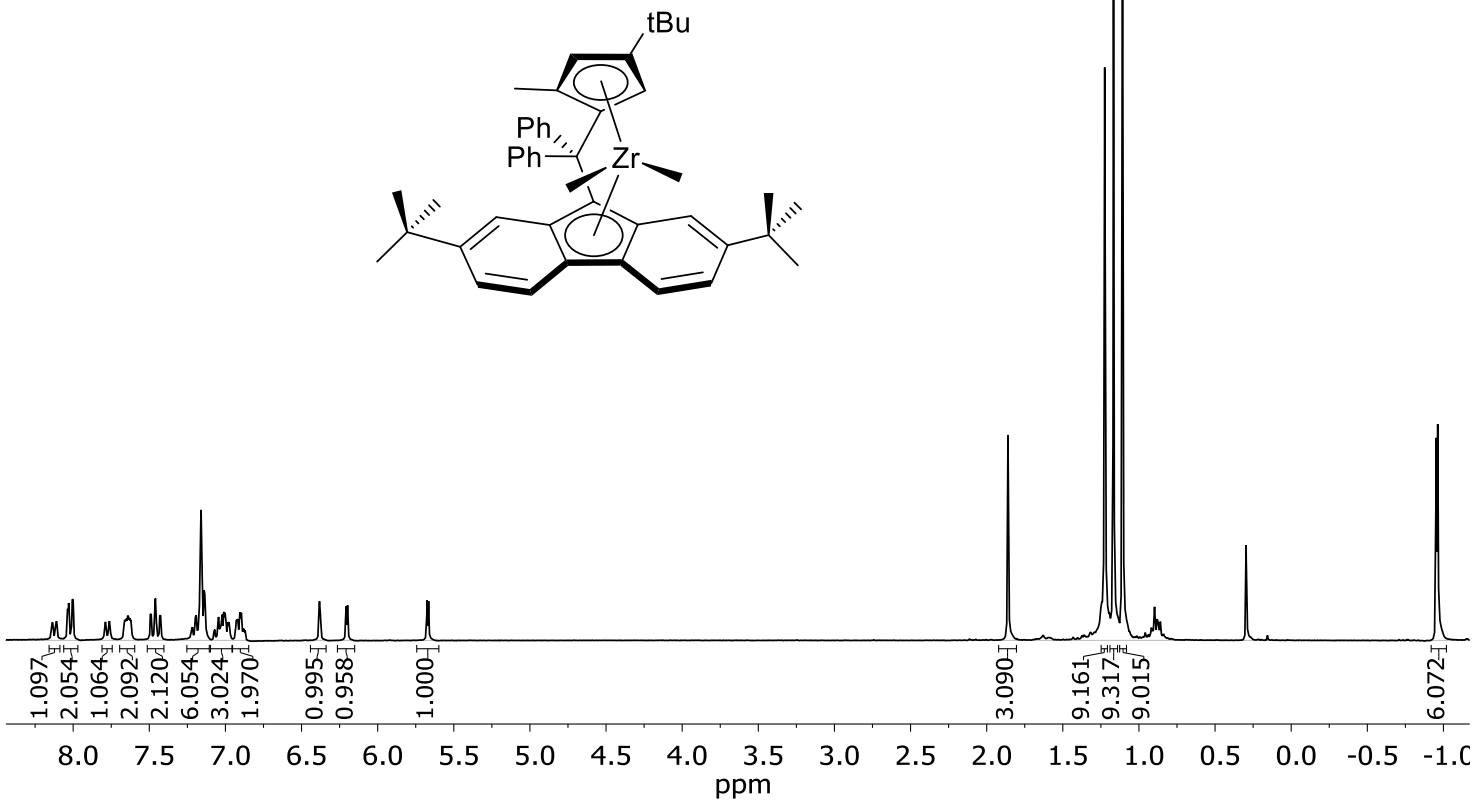

Figure S18. ${ }^{1} \mathrm{H}$ NMR spectrum $\left(\mathrm{C}_{6} \mathrm{D}_{6}, 400 \mathrm{MHz}, 25{ }^{\circ} \mathrm{C}\right)$ of $\{\mathbf{C p} / \mathbf{F l u}\}-3 \mathbf{a}$. 


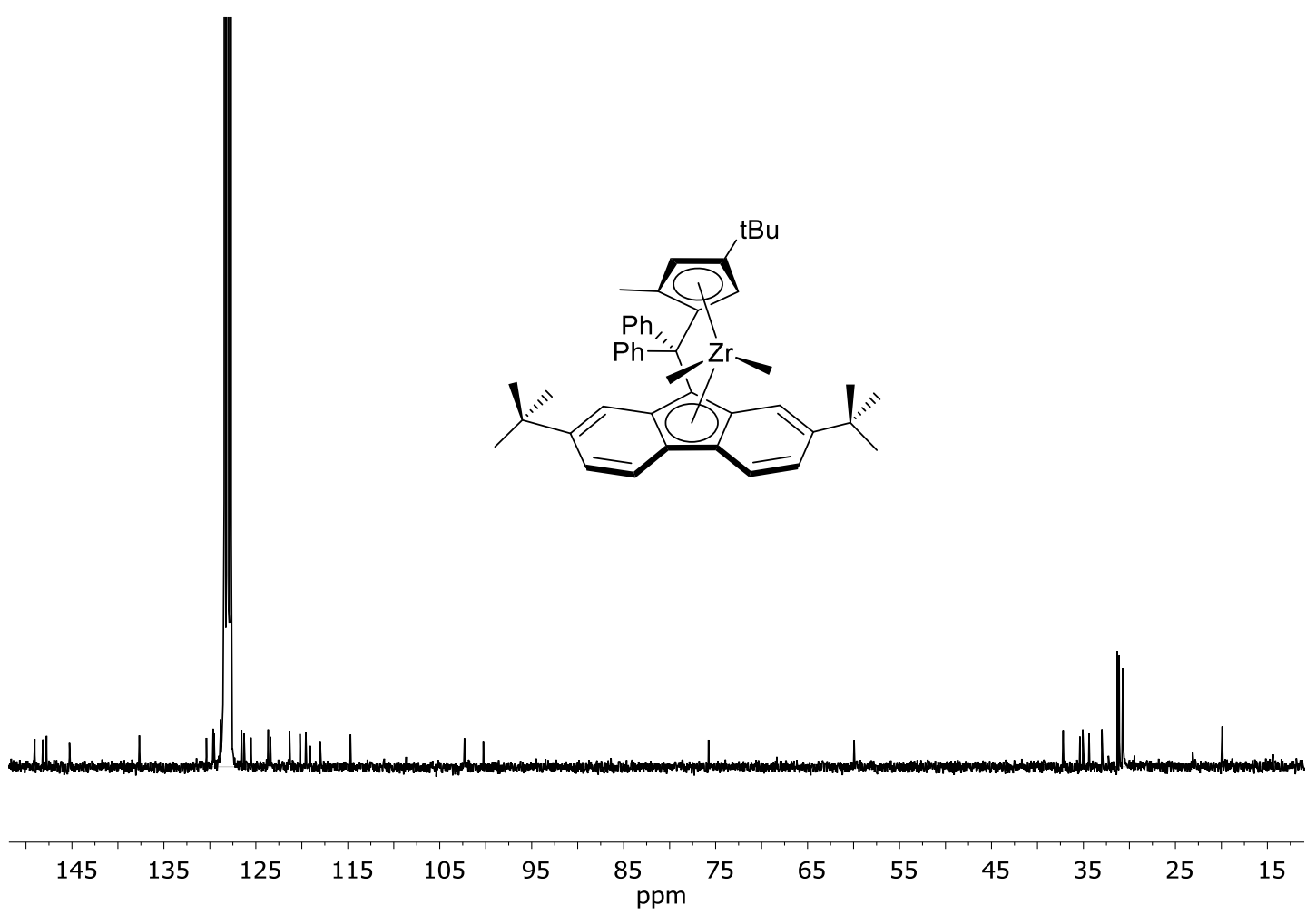

Figure S19. ${ }^{13} \mathrm{C}\left\{{ }^{1} \mathrm{H}\right\}$ NMR spectrum $\left(\mathrm{C}_{6} \mathrm{D}_{6}, 100 \mathrm{MHz}, 25^{\circ} \mathrm{C}\right)$ of $\{\mathbf{C p} / \mathbf{F l u}\}-3 \mathbf{a}$.

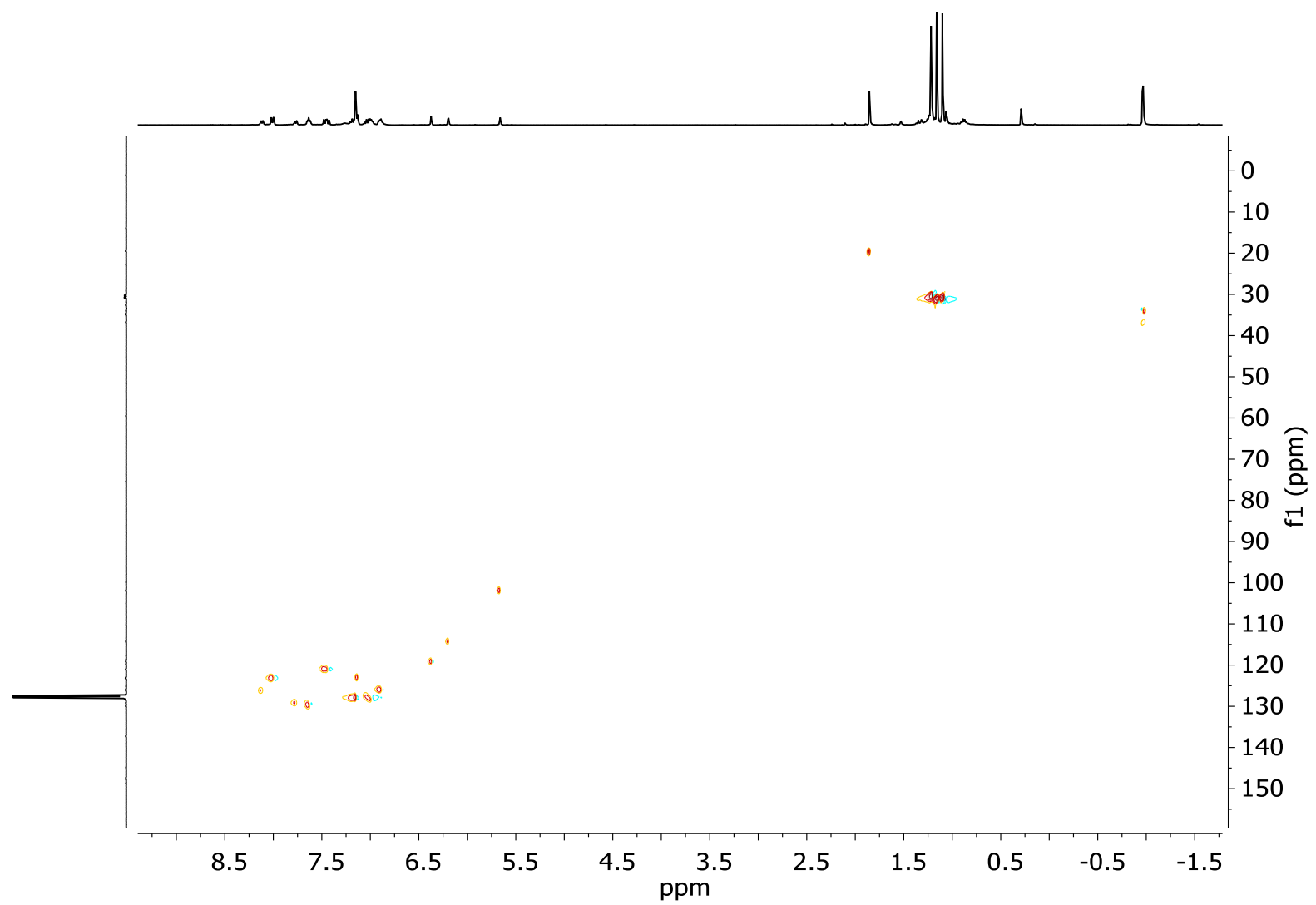

Figure S20. ${ }^{1} \mathrm{H}_{-}{ }^{13} \mathrm{C}$ HSQC NMR spectrum $\left(\mathrm{C}_{6} \mathrm{D}_{6}, 400 \mathrm{MHz}, 25^{\circ} \mathrm{C}\right)$ of $\{\mathbf{C p} / \mathbf{F l u}\}-3 \mathbf{a}$. 


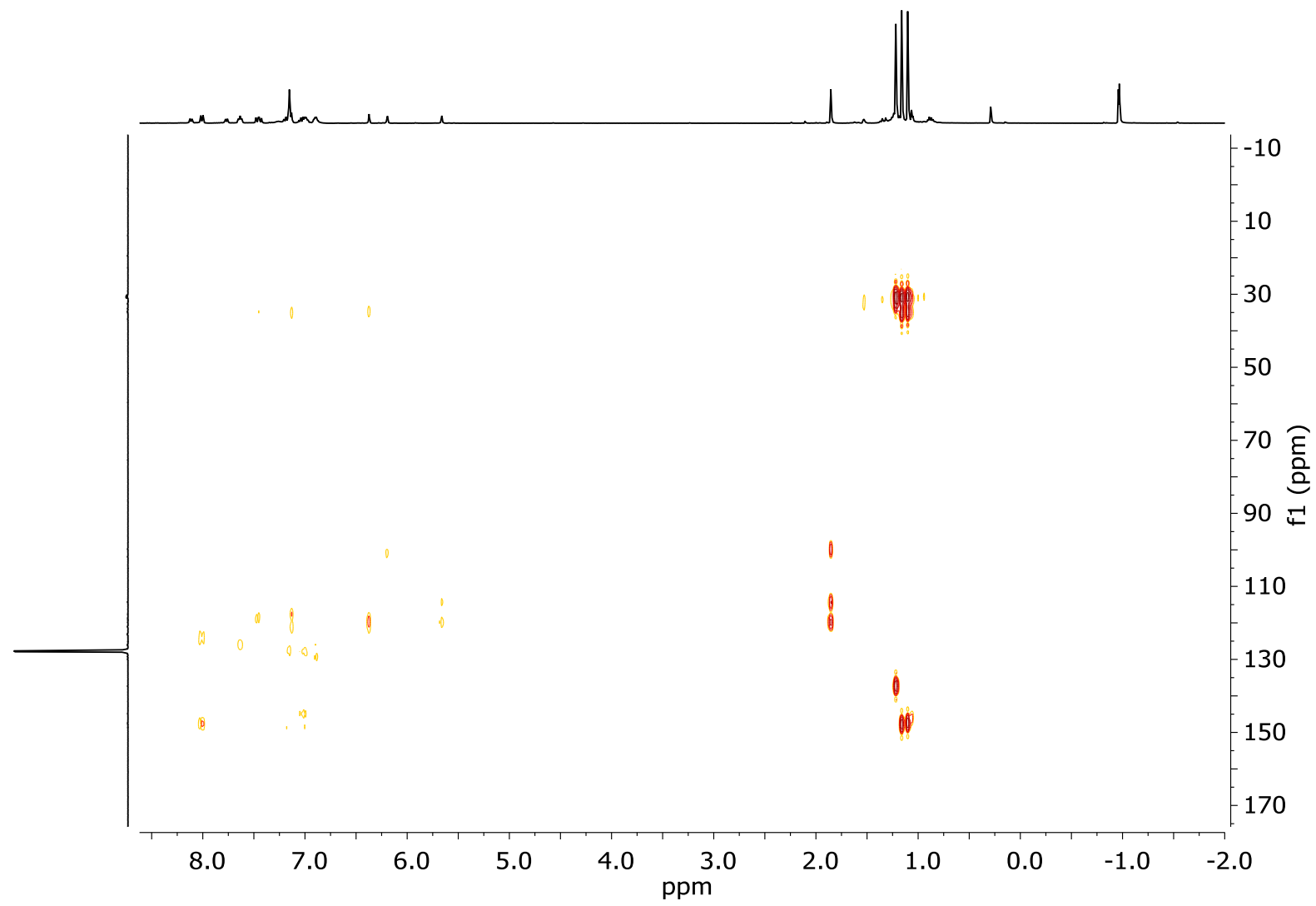

Figure S21. ${ }^{1} \mathrm{H}_{-}{ }^{13} \mathrm{C}$ HMBC NMR spectrum $\left(\mathrm{C}_{6} \mathrm{D}_{6}, 400 \mathrm{MHz}, 25{ }^{\circ} \mathrm{C}\right)$ of $\{\mathbf{C p} / \mathbf{F l u}\}-3 a$.

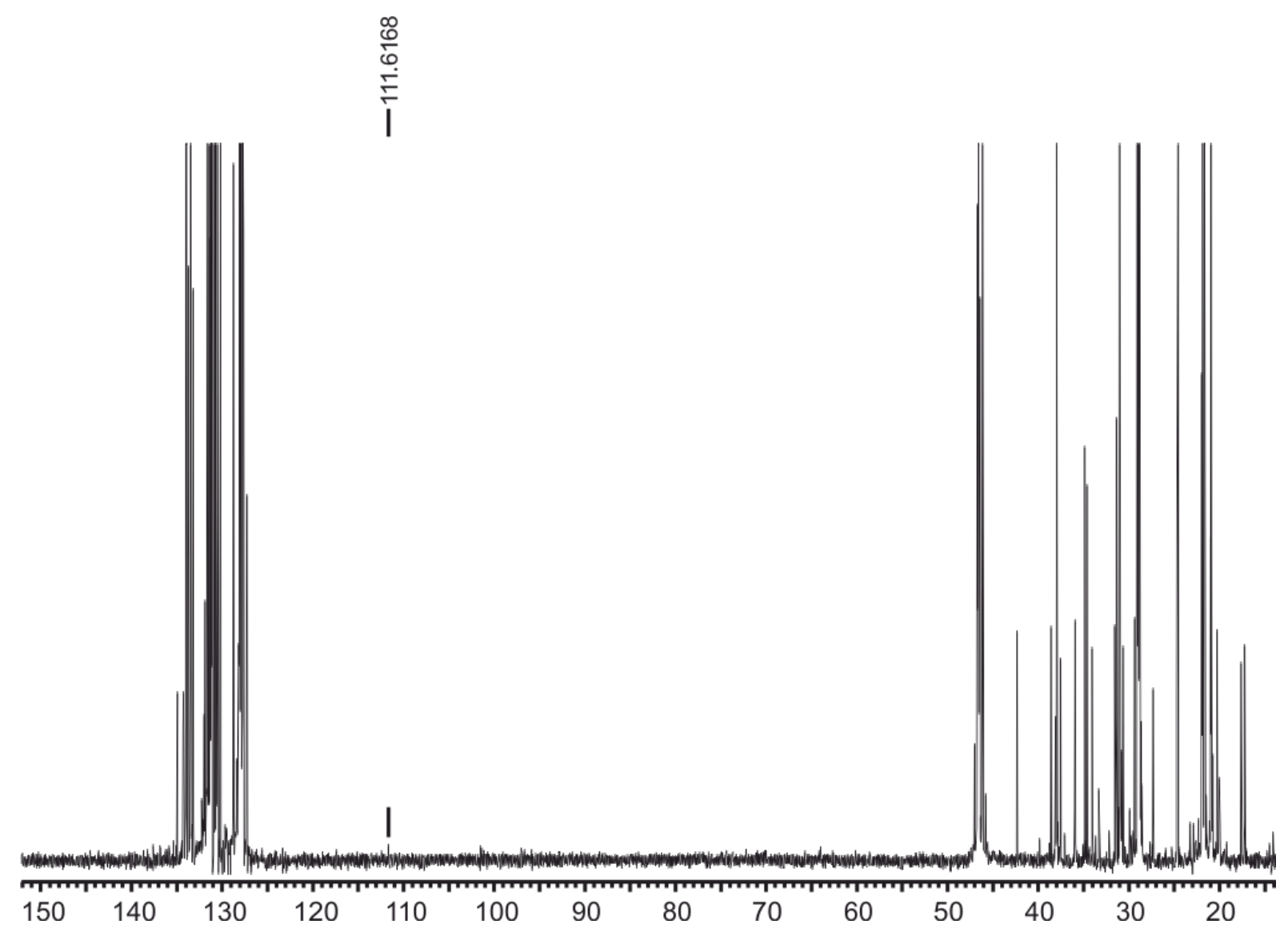

Figure S22. ${ }^{13} \mathrm{C}$ NMR spectrum $\left(125 \mathrm{MHz}, 135{ }^{\circ} \mathrm{C}, 1,2,4\right.$-trichlorobenzene/ $\left.\mathrm{C}_{6} \mathrm{D}_{6}\right)$ of copolymer obtained from propylene and ethylene with the $\{\mathbf{S B I}\}-2 / \mathrm{MAO}$ system. 


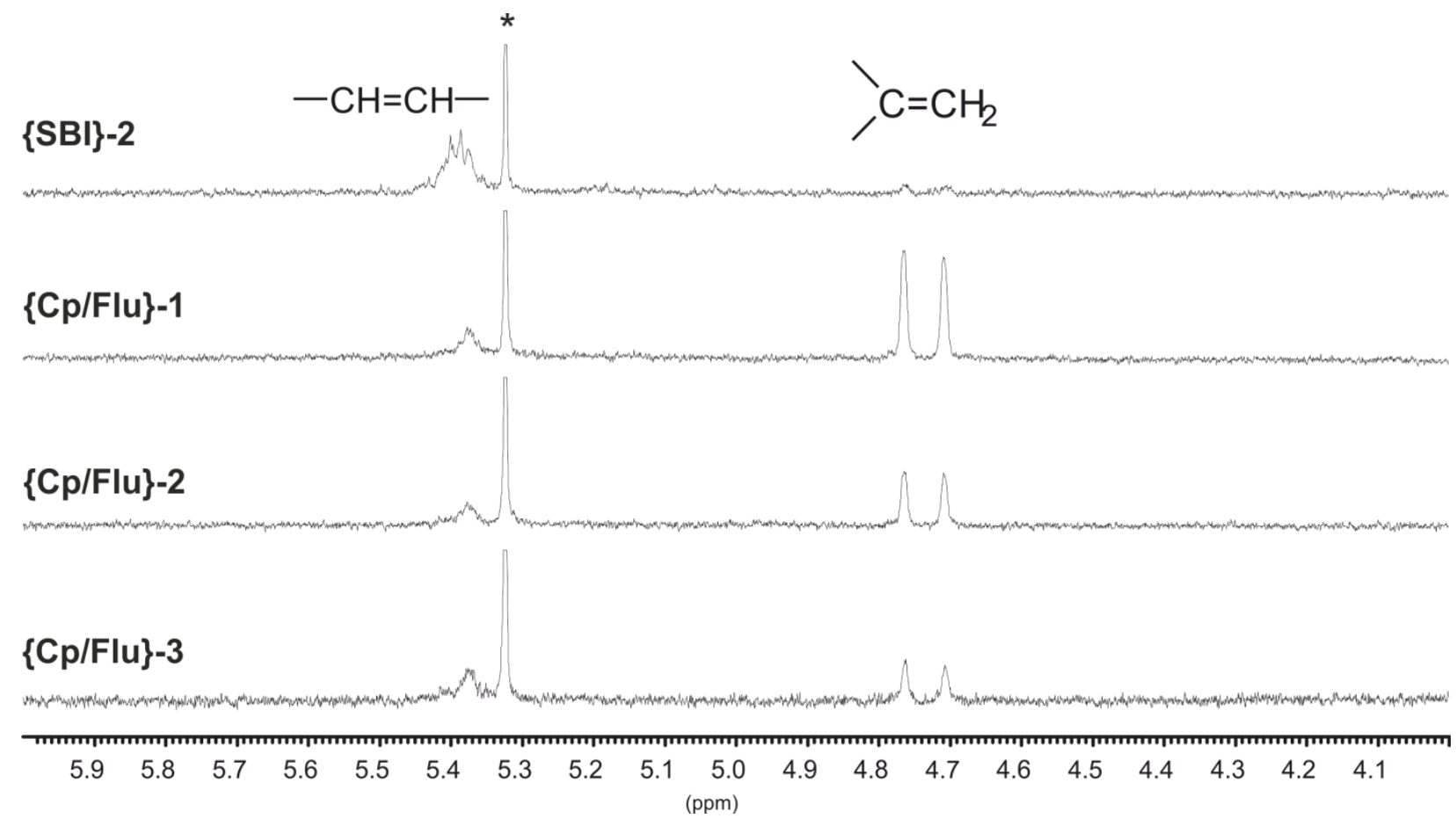

Figure S23. The end-groups region of ${ }^{1} \mathrm{H}$ NMR spectra $\left(400 \mathrm{MHz}, 25{ }^{\circ} \mathrm{C}, \mathrm{CDCl}_{3}\right)$ of poly $(1$ hexene)s obtained with metallocene/MAO systems in toluene (Table 2, entries 1, 3, 6 and 9, respectively). * stands for residual $\mathrm{CH}_{2} \mathrm{Cl}_{2}$. 